\title{
AUTHOR INDEX OF 37TH WORLD CONGRESS OF ENDOUROLOGY \\ by abstract number
}

A

ABABNEH, M, MP30-21

ABAZA, R, MP02-19, MP12-07, MP15-12, MP18-05, MP18-18, MP19-16

ABBARAJU, J, MP01-16

ABBAS, L, MP05-17

ABBOTT, JE, MP26-22, MP28-05, MP28-09

ABBOUDI, H, MP10-02, MP16-01, V02-05

ABBUD, N, MP06-13, MP06-17, MP11-16

ABDALLA, O, V18-08

ABDALLAH, M, MP03-01

ABDALLAH, N, MP08-14, V01-04

ABDEL-RAZZAK, O, MP12-08

ABDELDAIEM, H, MP29-22

ABDELHAKIM, M, MP01-20, MP12-08

ABDELSALAM, A, V15-12

ABDELSALAM, YM, MP07-22

ABDIMALIK, M, BSP2-02

ABDRAHIMOV, R, V08-08

ABDUL-MUHSIN, H, MP18-09

ABDULLAHOGLU, D, V06-02

ABDULRAHMAN, M, MP07-22

ABE, T, MP06-06, V10-03

ABE, Y, MP17-17

ABIAN, N, MP24-08

ABOUMARZOUK, OM, MP05-16, MP11-18, MP14-07, MP19-15, MP27-15, MP30-08

ABRAHAM, JA, MP13-14, MP28-08, MP28-20

ABUFARAJ, M, MP30-21

ABUZENADA, M, MP16-16

ACHER, P, MP09-21

ACQUATI, P, BSP2-16, MP03-04

ADAMIC, B, MP20-02

ADAMOU, C, MP03-06, MP23-13, MP26-03, MP30-03

ADASONLA, K, MP16-01

ADAWI, F, V03-01, V14-10, V15-06, V16-11

ADIYAT, K THEKKE, MP19-19

ADOT, J, MP24-06

AFERIN, U, V08-06, V14-03

AGARWAL, D, MP09-14, MP23-07

AGARWAL, K, MP05-03

AGARWAL, R, MP30-02

AGARWAL, S, MP28-13, MP30-02

AGARWAL, V, V06-06, V12-07

AGRAWAL, G, MP24-11, V01-01, V10-11

AGRAWAL, MS, MP28-21

AGRAWAL, S, MP10-08, MP25-17, V15-05

AHALLAL, Y, V17-05

AHLERING, TE, MP01-10, MP01-12, MP17-16

AHMED, I, MP05-15, MP14-02, MP16-19, MP22-05, MP26-08, MP27-03
AHMED, K, MP06-06, MP06-11, MP06-13, MP06-17, MP06-18, MP11-16, MP16-13, MP16-14, MP20-17 AHMED, M, BSP2-15, MP10-21, MP11-01, MP18-02,

MP18-13, MP18-17, V03-03, V08-10, V09-12, V13-09 AHN, J, MP06-16, MP20-05

AHSANUDDIN, S, V08-10, V13-09

AIZEN, J, MP20-02

AJAMI, T, V18-02, V18-11

AJAYI, L, MP02-12, MP22-18, MP23-12, MP23-16

AKATSUKA, J, MP03-11, MP05-19

AKDEMIR, S, MP08-20

AKGÜL, H, MP25-06

AKHTAR, A, MP29-01

AKHTER, S, MP07-14, MP27-01, MP28-02, MP29-15

AKOPYAN, G, MP10-17, MP30-05

AKPINAR, H, MP25-06

AKRASH, H Al, MP13-06

AL-HARBI, B, MP22-06, MP22-17

AL-HAYEK, S, MP21-01

AL-JABIR, A, MP06-06, MP06-11

AL-OMAR, O, V06-09

AL-RAMMAHI, D, MP07-15

AL-SALAM, S, MP04-10

AL-SOLUMANY, A, MP07-09, MP16-16

AL-TERKI, A, MP28-10

AL ZAHRANI, A, MP13-06, MP15-05

AL, K, MP04-17

ALABBAD, A, BSP2-06, BSP2-13

ALAHMARI, A, V15-12

ALALAO, O, MP20-18, MP26-01

ALALI, K, V12-09

ALAM, M, MP09-07

ALAMARI, W, BSP2-06, BSP2-13

ALAMIRI, J, MP08-03, V18-04

ALAMO, J FERNANDEZ DEL, MP03-18, V07-03

ALAMOUDI, T, V15-12

ALANBUKI, A, MP24-22

ALAQQAD, A, MP20-16, V05-11

ALASKER, A, MP26-17

ALBA, A BUDÍA, MP21-12

ALBANESI, L, MP16-05, V17-08

ALBAYRAK, A, BSP2-10, BSP2-17, MP03-03, MP23-02

ALBERTO, J, MP01-19

ALCAIDE, J RAMÓN CANSINO, MP06-06, MP21-12

ALCARAZ, A, MP14-10, MP18-08, V10-06, V18-02, V18-11

ALDHAAM, N.A., BSP1-10

ALDHAAM, NA, MP01-01, MP01-20, MP06-15, MP12-08,

MP15-09, MP15-23, MP20-08, V08-04

ALDOKHI, M, BSP2-06

ALDOUKHI, A, MP25-05, MP30-04, MP30-16

ALENEZI, A, MP12-13 
ALENEZI, N, MP21-08

ALENIZI, AM, V06-05, V11-02

ALENZI, M, MP17-15

ALEXANDROV, I, V06-08, V16-08

ALGAHWARI, M, BSP2-06, BSP2-13

ALGARNI, K, V15-12

ALGOSAIBI, A, V11-02

ALHAJERI, F, V12-09

ALHAMAD, M, V03-01, V14-10, V15-06

ALHARTHI, M, MP07-09, MP16-16

ALHAZMY, A, MP07-09, MP16-16

ALI, A, MP05-17

ALI, A., BSP1-12

ALI, B, MP18-10

ALI, M, MP07-21

ALI, S, MP10-17, MP30-05

ALI, Z, MP30-07

ALJAMAL, S, MP30-21

ALJNEIBI, A, V06-07

ALKHALILI, A, V03-01, V14-10, V15-06

ALKHAMEES, MA, MP26-17

ALKHUDIR, S, V12-09

ALLAF, M, MP14-01

ALLAHVERDIYEV, M, MP23-02

ALLEN, D, MP02-12, MP22-18

ALLEN, S, MP05-09

ALMALLAH, Z, MP20-16

ALMEIDA, HS, MP30-19

ALMERAS, C, MP25-09

ALMOUSA, R, BSP2-06, BSP2-13

ALMUJALLID, A, MP26-17

ALMULHIM, A, V11-02

ALMUSHATAT, A, MP30-07

ALNAJJAR, HM, MP16-13

ALOBAIRI, Y, MP16-16

ALOISI, P, MP16-05, V17-08

ALOTHMAN, A, MP26-17

ALOTHMAN, KI, MP02-02

ALOWIDAH, I, MP17-15

ALRABADI, A, MP30-21

ALSADHAN, M, MP26-17

ALSAWI, M, MP20-21

ALSHARIF, A, MP30-21

ALSHOHAYEB, I, MP26-17

ALSUNBUL, AA, MP15-05

ALSYOUF, M, MP04-08, MP14-06, MP21-05, MP21-22,

MP24-07, MP30-11

ALTHUNAYAN, AM, MP30-13

ALUR, JB, MP27-10

ALUR, SB, MP27-10

ALVAREZ, F. NATAL, BSP1-08, MP16-22, V17-03

ALWEHAIBI, A, MP02-02

ALY, S, V14-08

ALZAHRANI, T, MP13-06, MP15-05

ALZUGHABI, M, MP26-17

ALZWERI, LM, MP17-18

AMARO, J, MP26-07

AMASYALI, A, MP04-08, MP14-06, MP21-05, MP21-22, MP24-07, MP30-11

AMATO, M, MP08-18, MP26-02

AMBANI, SN, MP30-16

AMERI, C, MP08-19, MP14-05, MP26-06

AMIGONI, N., BSP1-14

AMIN, MS, MP06-13, MP06-17, MP11-16
AMPONIN, ME, MP28-20

AMSTER, M, MP14-09

AN, D, MP18-01, V06-04

AN, L, BSP1-22, MP04-16, MP05-18, MP07-11, MP07-12, MP11-10

ANACLETO, S, MP01-19, MP06-07, MP24-12, MP24-18, MP28-22

ANAN, G, MP12-10

ANASTOS, H, MP14-22

ANCESCHI, U, MP09-05, MP11-20, MP14-19, MP15-15, MP15-18, MP17-20, MP18-19, MP18-21, V08-11, V14-06

ANDERSON, P, MP03-10

ANDERSSON, S, MP07-15

ANDONIAN, S, MP05-14

ANDRÉN, O, MP07-15

ANDRADE, HS, MP13-22, V12-08

ANDURA, O, MP13-02, V05-03, V10-02

ANGERRI, O, V04-04

ANJUM, F, MP29-10, V18-08

ANTONELLI, A, MP13-01, MP13-09, MP18-21

ANTONELLI, JA, MP05-14, MP22-09, MP22-11,

MP22-16, MP29-01, MP29-16

ANTONUCCI, M, BSP1-18, MP11-17, V02-02

AOKI, M, MP03-17

AOWN, A Al, MP03-06

APOJ, M, MP02-15

APONTE, FA OQUENDO, MP03-22, V07-04

APRIKIAN, A, BSP2-05

ARAÚJO, GB, MP30-19

ARAFENIAN, S, MP11-11

ARAI, S, MP03-17

ARAKI, K, V03-04, V05-02

ARAP, MA, MP13-22, MP15-20, V12-08

ARBALAEZ, C MEJIA, MP23-12

ARGADE, SP, MP22-12

ARGUN, Ö, MP16-17, MP18-15

ARISTIZABAL, J, MP05-01

ARMAS-PHAN, M, MP07-04, MP23-18

ARNOUK, A, MP24-02

ARO, T, MP10-11, MP10-13, MP22-02

ARON, M, MP13-01, MP13-09

ARORA, K.A., BSP1-09

ARSLAN, M, MP08-20, MP15-07

ARTIBANI, W., BSP1-14

ARUMUHAM, V, MP05-09, MP10-02

ASGHAR, AM, MP09-19, MP17-10, V06-11, V11-10

ASKARI, S, MP23-17

ASLAM, Z, MP13-16

ASLAN, Y, MP08-20, MP15-07

ASPLIN, J, MP22-14

ASSIMOS, D, MP04-18, MP22-04, MP22-07

ASTROZA, GM, MP20-01

ATAGI, Y, V02-03, V04-01

ATALLAH, W, MP04-15, MP05-07, MP05-10, MP22-01, MP23-09, MP30-12, MP30-22

ATHER, H, BSP1-01, MP28-01, MP29-08, MP30-20

ATUĞ, F, V02-09, V03-10, V08-01, V11-08, V14-05

AU, W, V02-10

AUBE, M, BSP2-05, MP08-05, MP08-07

AUCIELLO, M, MP28-14, V18-07

AUM, J, MP18-01, V06-04

AUTORINO, R, MP13-01, MP13-09, MP18-21, V09-02, V11-05, V15-11 
AVERCH, T, MP07-19

ÁVILA, A, MP26-16, MP28-04

AWAD, M, MP01-14

AYANI, E, MP27-05

AYBAL, HC, V01-07

AYDIN, R, MP24-09

AYDIN, A, MP06-06, MP06-11, MP06-13, MP06-17, MP11-16, MP16-14

AYDIN, B, MP24-09

AYDOGAN, TB, V01-07

AYERS, B, MP15-06

AYKANAT, C, MP08-20

AYTAC, O, V02-09, V03-10, V08-01, V11-08, V14-05

AYUB, A, V10-01

AZAD, M, V10-01

AZHAR, R, V15-12

AZIZ, W, MP29-08

\section{B}

BABAWALE, O, MP14-07

BABAYAN, R, MP21-04

BABOUDJIAN, M, MP30-06

BACH, T, MP03-07, MP03-10

BADANI, KK, MP02-05, MP02-19, MP18-05, MP18-11, MP18-13, MP18-18, MP19-04, V01-08, V08-12, V09-03, V11-07, V14-07

BADAWY, A, MP21-08

BADHIWALA, N, MP06-22

BAE, W, MP17-01

BAEK, M, MP21-15

BAGLEY, D, MP10-07, MP27-02

BAIG, U, MP06-06

BAILLY, V, MP14-13

BAK, D, MP24-17

BALAÑ்̀, J, V04-04

BALAH, A, MP16-09

BALAN, G, MP25-08

BALBAY, MD, V08-09

BALCI, M, MP08-20

BALDWIN, D, MP04-08, MP14-06, MP21-05, MP21-22, MP24-07, MP30-11

BALTHAZAR, A, V11-05

BALZARRO, M., BSP1-14

BAMBERGER, JN, MP04-15, MP05-07, MP05-10, MP22-01, MP23-09, MP23-14, MP23-15, MP28-14, MP30-12, MP30-22

BANDO, H, V02-03

BANERJEE, S, MP01-06

BANG, W, MP02-11

BANGASH, M, BSP1-01, MP25-02, MP29-04

BANGMA, C, MP19-09

BANSAL, D, BSP2-12

BAO, G, MP01-03, MP01-08, MP21-06

BAPTISTUSSI, M, MP26-07

BARAYAN, G, MP01-14

BARBER, NJ, MP02-20, MP03-09, V01-11

BARNARD, JT, V06-09

BARON, F ROUSAUD, MP22-21

BARR, A, MP27-15

BARRETTO, N, V03-06

BARRETTO, NJ, V03-06, V11-03

BARROS, AA, MP16-08, V17-10

BARTHE, F, V17-05
BASATAC, C, MP25-06

BASHAR, A, MP29-17

BASHETTY, RS, MP27-10

BASHORUN, T, MP19-01

BASRALIAN, K, MP14-14, V13-08

BASTIANPILLIAI, C, MP25-04

BASULTO, M, MP28-04

BATAGELLO, C, MP07-04

BATEH, R, MP17-02

BATTER, T, MP07-04

BAYKOV, N, V02-06, V17-04, V17-09

BECHIS, S, MP09-16, MP24-01, MP26-11

BECKER, B, MP11-15, MP29-03

BEKSAC, A, MP02-19, MP18-05, MP18-11, MP18-18, MP19-01, V01-08, V08-12, V09-03, V11-07, V14-07

BELAY, R, MP21-22, MP30-11

BELFAST, E, V06-03

BELKOVSKY, M, MP11-05, MP17-04, MP17-05

BELL, C, BSP2-02, MP03-13

BELLINZONI, P, MP02-13, V18-10

BENEDETTO, E Di, MP03-08

BENJAMIN, T, MP28-16

BENSADOUN, H, MP16-10, MP30-09

BERDONDINI, E, MP08-09

BERGE, V, MP12-22

BERKER, D, MP15-07

BERNARDO, N, MP26-07

BERNHARD, J, MP16-10, MP30-09

BERTHE, L, MP04-06, MP04-09, MP29-12

BERTOLO, R, MP11-20, MP18-21

BESANA, U, MP01-15, MP03-12, MP06-08, MP07-10,

MP09-12, MP14-17, MP21-03, MP25-13

BESOMBES, T, MP10-14

BEST, SL, MP06-02, MP22-09, MP22-11, MP22-16

BEULENS, A, MP19-09

BEVILACQUA, L, MP04-13, MP26-02, MP27-17

BHAMBRA, T, MP02-16

BHANDARI, A, MP02-19, MP18-05, MP18-18

BHAT, S, MP06-10, MP12-05, MP12-11, MP12-19, MP17-12, V03-02, V03-07

BHATIA, S, MP13-21

BHATTACHARYYA, MK, MP08-16

BHATTI, K, V18-06

BHATTY, T, MP07-14, MP27-01

BHIRUD, P, MP03-16, MP11-12, V02-12, V07-06,

V07-11, V07-12, V10-05

BHOJANI, N, MP03-10, MP08-11

BIANCHI, G, MP04-13, MP16-11, MP27-17

BIDAD, R, MP24-17

BIEBEL, M, MP21-04

BIGLEY, J, MP07-19

BILAL, KH, MP11-09, MP20-14

BILAL, S, MP10-02

BILIGERE, S, MP29-02

BILLAH, M, BSP2-15, MP18-13, MP18-17, V08-10,

V13-09, V13-10, V15-04

BILLOO, Z, MP13-08

BINGFENG, L, MP23-10

BIRD, VG, MP05-14

BIRKS, T, BSP1-07, MP02-12, MP23-12, MP23-16

BIYANI, S, MP02-16

BJAZEVIC, J, MP04-11, MP04-17, MP24-04

BLACH, O, MP24-22

BLACK, K, MP25-05, MP30-04, MP30-16 
BLADOU, F, MP16-10, MP30-09

BLAS, L, MP08-19, MP14-05

BLEZIEN, O, BSP2-16, MP03-04

BLUEBOND-LANGNER, R, V13-03

BOGARD, D, MP20-15

BOGOMOL, A, MP14-14

BOISSIER, R, BSP1-16, MP01-02, MP16-20, MP18-08,

MP19-20, MP23-20, MP30-06, V02-08

BOMANJI, J, MP05-09

BONANNO, N, MP08-19, MP26-06

BONI, A, MP27-18

BOONYAPANICHSKUL, P, MP02-22

BORGES, R, MP18-04

BOSE, P, MP02-04, MP02-21, MP20-19

BOTHA, A, BSP2-19

BOYD, C, MP04-18, MP22-04, MP22-07

BOYDSTON, K, MP04-22, MP21-20

BOYKO, A, MP21-04

BOYLU, U, MP17-09

BOZZINI, G, MP01-15, MP03-12, MP06-08, MP07-10, MP09-12, MP14-17, MP21-03, MP25-13

BRASIL, TM, MP30-19

BRASSETTI, A, MP11-20, MP14-19, MP15-15, MP15-18, MP17-20, MP18-19, MP18-21, V08-11, V14-06

BRAVO-CORDERO, J., BSP1-02

BREDA, A, BSP1-16, MP01-02, MP16-20, MP18-08, MP19-20, MP23-16, V02-08

BREISH, MO, MP01-17, MP29-10, V05-07, V18-08

BRENNHOVD, B, MP12-22

BREVIK, A, MP03-19, MP08-12, MP08-15

BREWIN, J, MP06-11

BRIGANTI, A, MP05-06, MP29-19

BRINKMAN, JE, MP04-12

BRINKMAN, W, MP19-09

BRITTAIN, J, MP20-04

BRODY, R., BSP1-02

BROGGINI, P, MP03-12, MP14-17

BROOME, J, MP05-03

BROUWERS, T, MP06-11

BROWN, C, MP05-10

BROWNE, D, MP29-10

BRUNCKHORST, O, MP06-11, MP16-13

BRUNKE-REESE, D, MP22-13

BRYANT, T, MP14-21

BUCKLEY, J, MP09-16

BUCUR, C, MP25-21

BUIZZA, C, MP01-15, MP03-12, MP06-08, MP07-10, MP09-12, MP14-17, MP21-03, MP25-13

BULBUL, E, V02-04, V06-02

BULBUL, M, MP08-14, MP13-18, V01-04

BURHAM, A, MP20-16

BURNS, H, MP07-08, MP20-21, MP26-18

BURTON, J, MP04-17

BUSTROS, G, V01-04

BYUN, H, MP26-09, MP29-07

C

CABAL, R., BSP1-02

CABRERA, JD, MP04-19, MP07-18, MP22-22

CABUNGCAL, A, MP07-05

CACACCIO, J., BSP1-10, BSP1-19

CADEDDU, JA, MP13-10, MP14-11, V09-07, V13-01

CALÓ, B, MP01-19
CALAZANS, LM, V03-06, V11-03

CALLAS, GA, MP26-03, MP30-03

CALORI, A, MP09-12, MP14-17, MP21-03, MP25-13

CALVERT, R, MP05-03

CALVO-VAZQUEZ, I, MP25-10, V12-11

CALVO, N, MP25-18

CAMARGO, CP, MP13-22

CAMPBELL, T, V06-03

CAMPI, R, MP18-08

CAMPOS, J, MP26-07

CAMPWALA, Z, MP14-06, MP21-22

CANDA, AE, V08-09

CANFIELD, S, MP14-16

CANTELLANO-OROZCO, M, MP07-20, MP25-10, V12-11

CANUL, S, MP28-04

CANUTO, GF, V11-03

CANVASSER, NE, MP05-14, MP10-04

CAO, D., BSP1-20

CAPITANIO, U, MP13-01, MP13-09, MP18-21

CAPON, G, MP16-10, MP30-09

CARBONE, A., BSP1-14

CARDENAZ-ORTIZ, A, MP26-07

CARLOS, E, MP04-22, MP21-20

CARMIGNANI, L, BSP2-16, MP03-04

CARR, C, MP19-18

CARRIER, S, BSP2-05, MP08-05, MP08-07

CARRIERI, G, MP02-01, MP28-14, V18-07

CARRION, RJ VALERO, MP15-13, V09-08, V13-07, V13-12

CARROCI, K, MP01-01

CARVALHO, AA, MP30-19

CARVALHO, N, MP06-07

CARVALHO, P, MP15-20

CASELLATO, S, BSP2-16, MP03-04

CASTELLANA, J CUÑÉ, MP21-12, MP22-21

CASTELLANOS, E, MP05-01

CASTILLO, G, V10-09

CASTLE, E, MP18-09

CATARINICCHIA, SP, MP19-12

CATTERWELL, R, MP03-08

CECCARELLI, P, MP27-17

CELENTANO, G, MP10-02, MP16-01, V02-05

CELIK, O, MP25-20

CELJAR, CM, MP30-19

CERESOLI, F, MP08-21, V01-10

CHABERT, C, MP02-20

CHADE, D, MP15-20

CHALLACOMBE, B, MP03-08, MP13-01, MP13-09

CHALOUHY, C, MP23-19

CHAMANIA, C, MP13-21

CHAN, L, MP04-04

CHAN, T, V02-10

CHANDHOKE, RA, MP04-15, MP05-07, MP05-10,

MP22-01, MP23-09, MP23-15, MP30-12, MP30-22

CHANDRA, D., BSP1-19

CHANG, C, V09-01

CHANG, H, MP09-09

CHANG, J, MP26-12

CHANG, K, MP17-15

CHANG, T, BSP2-01, V07-09

CHANG, Y, MP13-04

CHARITOPOULOS, K, MP02-18

CHAVALI, J, V17-06

CHEMALSÉ, C, V17-01 
CHEN, C, MP22-19

CHEN, G, BSP2-18, V09-01, V16-09

CHEN, H, MP23-03, MP25-22

CHEN, J, MP06-04

CHEN, K, MP06-14, V02-01

CHEN, L, MP13-03, V15-09

CHEN, M, MP26-19, V09-06, V16-01

CHEN, Y, MP09-22, MP23-03, MP25-22

CHEN, Z, MP27-16

CHENEY, S.M., BSP1-09

CHENG, L, MP04-21

CHEON, S, MP13-07

CHERUKU, R.R., BSP1-10, BSP1-19

CHESNUT, G, V08-03

CHETWOOD, A., BSP1-12

CHEVALLIER, D, V17-05

CHEW, BH, MP04-20, MP05-14, MP10-05, MP10-07, MP10-09, MP10-16, MP11-02, MP11-06, MP21-19, MP23-07

CHI, T, MP05-14, MP07-04, MP20-05, MP23-18, V04-13, V05-05

CHIA, N, V02-10

CHICA, A, MP05-01

CHIN, P, MP02-20

CHINENOV, D, MP30-05

CHIURA, A, MP10-07

CHO, D, MP09-08

CHO, H, MP17-01

CHO, J, MP02-11, MP09-08

CHO, S, MP24-07

CHOE, H, MP18-07, MP23-05

CHOH, N, MP27-09

CHOI, H, MP08-02

CHOI, J, MP09-08, MP21-15, MP26-09, MP26-12

CHOI, J., BSP1-06

CHOI, Y, MP17-15, V13-06

CHONG, J, MP05-10, MP06-18, MP19-03

CHOONG, S, MP10-02, MP16-01, V02-05

CHOTIKAWANICH, E, MP14-12, MP16-07

CHOU, C, MP09-09

CHOUDHARY, Gr, V12-13

CHOW, AK, MP05-05, MP11-11, MP19-13

CHOWDHURY, S, MP07-21

CHUA, WJ, MP03-20

CHUGH, S, MP27-07, MP27-15, MP30-17

CHUGHTAI, B, MP03-10

CHUNG, H, MP13-04

CHUNG, J, MP26-09

CHUNG, J., BSP1-11

CHUNG, T, V02-10

CIARLARIELLO, S, MP04-13, MP08-18, MP16-11

CICCARIELLO, M, MP14-19

CITGEZ, S, V02-04, V06-02, V08-05

ÇITGEZ, S, MP25-06

CIVITELLA, A, V16-07

CLAYMAN, RV, MP05-14, MP10-20, MP11-14, MP14-01, MP16-03, MP21-07

CLORELY, J, MP14-20

CLOUTIER, J, MP21-21, MP25-07, MP25-14

COBB, K, MP10-04

COCA, S, MP04-04

COELHO, R, MP15-20

COHEN, D, BSP1-07, MP09-21

COLLIE, J, MP21-01
COLLURA, D, MP08-09

CONDE REDONDO, C, BSP1-08, V01-12, V03-12,

V12-05, V16-06

CONNOR, MJ, MP20-04

CONROY, D, MP22-13

CONTRERAS, A, MP05-01

CONTRERAS, PN, MP08-19, MP14-05, MP26-06

COOK, P, MP05-21

COOPER, V, MP10-20

CORDEIRO, A, MP01-19

CORDEIRO, M, MP15-20

CORDES, J, MP01-18, MP29-06

CORMIO, L, MP02-01, MP28-14, V18-07

CORREIA-PINTO, J, MP06-07, MP16-08, V17-10

CORREIA, J, MP18-04

CORTES-RAYGOZA, P, MP07-20, MP25-10, V12-11

CORTI, S, MP08-21, V01-10

COSCIONE, A, MP09-21

COSTA-BAUZÁ, A, MP21-12

COSTA, M, MP06-07

COSTANTINI, M, MP11-20, MP18-19, V14-06

COTTA, B, MP24-01

COTTONE, C, MP16-03, MP21-07, V02-11

COVAS MOSCHOVAS, M, MP06-10, MP12-11, MP12-05,

MP12-19, MP15-20, MP17-12, V03-02, V03-07

COZIER, Y, MP20-13

COZZI, P, MP02-20

CRACCO, C, MP07-04

CRIGGER, C, MP05-08, V15-08

CRISTALLO, C, MP23-12

CRIVELLARO, S, MP17-14, MP17-19

CRIVELLI, J, MP29-01

CRUZ, E, MP26-16, MP28-04

CUMBY, C, MP02-12

CURRY, D, MP08-04

CWACH, K, MP20-03

CYNK, M, MP09-02

\section{D}

D'ANNA, M, V10-06

D'AMICO, M, MP20-13

D'ANCONA, F, MP07-03

DA CRUZ, J, MP11-05, MP17-04, MP17-05

DA SILVA, L, MP30-07

DA SILVA, G EUFRASIO, V12-08

DAELS, P, MP23-12

DAFOE, D, MP11-08

DALKIN, B, MP06-16

DANILKO, K., BSP1-21

DARCQ, C, MP14-13

DARRAD, M, MP28-10

DAS, N, MP14-02

DAS, R, MP03-14

DASGUPTA, P, MP06-06, MP06-11, MP06-13, MP06-17,

MP06-18, MP11-16, MP16-14, MP20-17

DASGUPTA, R, MP20-04

DAUDON, M, MP21-21, MP25-09

DAUW, CA, MP20-07, MP30-16

DAVALOS, JG, MP26-22, MP28-05, MP28-09

DAVENPORT, M, MP14-16

DAVID, R, MP02-21, MP20-19

DAVIES, R, MP14-20

DAVIS, J, MP22-05 
DAVIS, L, MP05-09

DAYAL, BD, MP02-19, MP18-05, MP18-11, MP18-18, V01-08, V08-12, V09-03, V11-07, V14-07

DAZA, J, MP18-05, MP18-11

DE CASTRO OLMEDO, C., BSP1-08

DE DOMINICIS, M, BSP1-18, MP11-17, V02-02

DE LEON, A DIAZ, MP14-11

DE NUNZIO, C., BSP1-14

DE OLIVEIRA, JB, V11-03

DE SOUZA, J, MP20-21

DEAN, S, MP09-01

DEANE, L, V04-05

DECAESTECKER, K, MP18-08

DECLUE, A, MP06-22

DEFIDIO, L, BSP1-18, MP11-17, V02-02

DEGEN, M, MP10-21, MP11-01, V03-05

DEL ZINGARO, M, MP27-18

DELANEY, D, MP20-16

DELL'OGLIO, P, MP18-21

DELMONTE, A, MP07-19

DEMEY, A, V03-01, V16-11

DEMIRDAG, C, V08-05, V08-06

DEMOUR, S Al, MP30-21

DENEYKO, A, V08-07, V08-08

DEOLANKAR, JC, BSP2-15, MP18-02, MP18-13, MP18-17, V13-10, V15-04

DEQIANG, G, MP10-15

DERWEESH, I, MP13-01, MP13-09, MP18-21

DESAI, A, MP16-12, MP22-12

DESAI, M, BSP2-03, MP10-08, MP19-10, MP25-17, MP26-20, V15-05

DESAI, S, MP14-09

DESCOTES, J, MP17-20

DESROCHES, B, MP10-21, MP11-01, V03-05, V13-08, V14-08

DIAB, N, MP18-02

DIAMOND, S, MP14-16

DIANATNEJAD, S, MP20-11

DIAS, AT, MP30-19

DIAS, E, MP01-19, MP06-07, MP16-08, MP24-18, V17-10

DIAZ, T, MP28-04

DÍAZ ROMERO, J, BSP1-08, MP16-22, V17-03

DIEZ, J, V01-12

DILENA, R, MP22-06, MP22-17

DING, J, MP24-16

DIPINA, T, MP09-16

DISSELHORST, GW, MP04-03

DOĞANCA, TS, MP16-17, MP18-15

DO, R, MP04-04

DOBBS, RW, MP01-13, MP12-04, MP17-14, MP17-19, MP20-20

DOHERTY, S, BSP2-19

DOIZI, S, MP04-06, MP04-09, MP05-22, MP10-14, MP20-06,

MP21-21, MP27-05, MP29-12, MP29-19

DOKANIA, K, BSP2-22

DOLEK, B, MP24-21

DOPPALAPUDI, SK, MP14-16, MP14-22

DORA, C, V07-07

DORE, K, MP06-09

DORRELL, J, MP05-15, MP20-10, MP27-03, MP30-01

DOS SANTOS, CP, V11-03

DOU, K, MP06-04

DROLET, R, MP25-07

DU, K, MP06-22, MP16-12, MP18-16
DU, Y, MP06-04

DUARTE, C, MP05-01

DUARTE, RJ, MP13-22, V12-08

DUNNE, MM, MP26-22, MP28-05, MP28-09

DURAND, M, BSP1-02, BSP1-17, V17-05

DURRANI, F.A., BSP1-10, BSP1-19

DURRANI, M, MP15-09, V08-04

DURRANT, J, MP21-01

DY, GW, V11-06, V13-03, V13-05

DYMKOV, I, V06-08, V16-08

DYMOV, A, MP01-04, MP10-17, MP30-05

E

EBAID, G, MP15-20

EDDY, BA, MP06-19, MP14-02, MP14-04, MP14-08, MP14-15, MP16-19, MP17-03, MP17-07, MP18-12, MP19-03, MP20-10, MP20-15, V11-12

EDELSON, M, MP02-14

EDEN, C, MP12-13

EDISON, M, MP20-04

EFTHIMIOU, I, MP02-14

EISNER, B, MP02-13, MP04-19, MP07-04, MP10-08, MP23-12, V18-10

EISSA, A, MP04-13, MP16-11, MP27-17

EJAZ, M, V10-01

EKBAL, S, MP08-06

EKWUEME, K, V16-05

EL-ASMAR, J, MP13-18, V01-04

EL-BAHNASY, A, MP17-15

EL-HUSSEINY, T, MP09-01, MP20-04

EL-KHOULY, M., BSP1-19

EL-LEITHY, T, MP05-17

EL-NAHAS, AR, MP28-10

EL-THOLOTH, H, MP13-06, MP15-05

ELBAKRY, AA, MP02-19, MP18-05, MP18-11, MP18-18, V01-08, V08-12, V09-03, V11-07, V14-07

ELBARONI, W, MP13-16

ELBERS, J REINOSO, MP03-18, V07-03

ELDRED, N, MP01-13

ELGANAINY, EO, MP07-22

ELGEBALY, O, MP16-09, MP29-22

ELGHARABAWY, M, MP21-08

ELHAGE, O, MP03-08

ELHALAWATY, O, MP30-07

ELHILALI, M, BSP2-05

ELKOUSHY, M, MP08-05

ELLA-TONGWIIS, P., BSP1-05

ELLIOTT, A, MP01-17

ELLIS, G, MP02-12, MP03-13

ELMANSY, H, MP03-22, MP08-07, V07-04

ELSAMRA, SE, MP19-21, V08-02, V10-08

ELSAYED, AS, MP01-01, MP01-20, MP12-08, MP15-09, MP15-23, MP20-08, V08-04

ELSHERBINY, A, MP04-13, MP27-17

ELTEMAMY, M, MP15-13

ELTERMAN, D, MP03-10

EMARA, A, MP08-01, MP08-13

EMILIANI, E, MP04-19, MP07-04, MP21-12,

MP22-21, MP22-22, MP23-16, MP30-08, V04-04

EMMERLING, M, V01-09

ENDO, F, MP09-06, MP12-02

ENDO, Y, MP03-11, MP05-19

ENG, S, MP24-05 
ENIKEEV, D, MP01-04, MP04-14, MP10-17

ENIKEEV, M, MP04-14, MP10-17

ENOKIDA, H, MP15-22

EPSTEN, M, MP05-05

ERÖZENCI, A, V08-05, V14-03

ERAIBEY, M, MP14-08

ERCILI, B, V02-04, V06-02, V08-05, V08-06, V14-03

ERGAKOV, D, V02-06, V17-04, V17-09

ERI, L, MP12-22

ERKAN, A, MP15-07

ERTEM, M, V08-06

ESCOBAR, M, V18-12

ESCUDERO, J. Calleja, BSP1-08

ESLAHI, A, MP07-13

ESPOSITO, M, V03-03

ESQUEDA, A, MP26-16, MP28-04

ESTRADE, V, MP16-10, MP25-09, MP30-09

ETEMADIAN, M, MP24-08

ETO, M, MP11-13

EUN, DD, BSP2-15, MP02-19, MP09-19, MP13-01, MP13-09, MP17-10, MP18-05, MP18-18, V06-11, V11-10

EURE, G, MP02-20

EVANS, M, MP13-16

EWALD, J, MP14-06

EYE, OV, V16-04

EZER, M, MP02-06

F

FADEL, M, MP03-14

FAISAL, F, MP20-22

FAKHOURI, F, MP11-05, MP17-04

FAKHRALDIN, SS, MP07-02, MP29-18

FALAGARIO, U, BSP1-02, BSP1-17, MP01-19, MP02-01, MP19-01, MP28-14, V17-05, V18-07

FALLARA, G, MP05-06

FAN, Y, MP13-04

FANG, J, V16-09

FARAG, F, MP08-04

FARAHAT, Y, MP06-06

FARAJ, K.S., BSP1-09

FARBER, NJ, MP19-21

FAREED, K, V13-02, V13-04, V13-11

FARGANOV, A, BSP2-21

FARHAN, M, MP28-01

FARIA, L, MP11-05

FARMER, T, MP08-01, MP08-13

FARRAJ, K, MP18-09

FARSARI, E, MP26-03, MP30-03

FAZOLI, A, MP15-20

FEGHALI, J, MP25-03

FEICKE, A, V11-11

FENG, A, MP10-15

FERNANDES, E, MP26-04

FERNANDES, V, MP06-07, MP24-18

FERNANDEZ-CAMILO, MA, V10-09

FERNANDEZ-NOYOLA, G, MP07-20, MP25-10, V12-11

FERNANDEZ, N, MP05-01

FERNANDO, H, MP25-04

FERRAZ, RF, MP30-19

FERREIRO, C, MP23-16

FERRER, O MAYORDOMO, V02-08

FERRIERE, J, MP16-10, MP30-09
FERRIERO, M, MP09-05, MP11-20, MP14-19, MP15-15, MP15-18, MP17-20, MP18-19,

MP18-21, V08-11, V14-06

FEU, O ANGERRI, MP07-04, MP21-12, MP22-21, MP23-16

FEYERABEND, B, MP11-15

FIALA, R, MP08-04

FIDANZA, F, MP04-13

FIGENSHAU, R, BSP1-20, MP06-22, MP16-12,

MP18-16, MP19-13

FIKRET, O, MP12-05, MP12-19, MP17-12, V03-02, V03-07

FINKELSTEIN, M, MP11-09, MP18-03, MP20-14

FLAMMIA, R, MP11-20, MP15-15, MP15-18,

MP17-20, MP18-19, V08-11, V14-06

FLEMING, C, V18-04

FLOR, J, MP07-05, MP28-06

FLORES, J, MP28-04

FOGG, L, MP05-05

FOLKARD, SS, BSP2-11, MP01-06

FOMBONA, A, MP30-14

FORBES, C, MP23-07

FORNARA, P, MP19-20

FORTE, V, MP14-19

FORTES, MQ, MP30-19

FOSSA, S, MP12-22

FRANCIS, MA DINNO, MP13-14

FRASCHERI, F, MP26-06

FREEDMAN, S, MP02-20

FREIXEDAS, F GRASES, MP21-12

FRIEL, B, MP30-14

FRIMBERGER, D, V06-01

FRYE, TP, V03-11, V15-07

FU, B, MP12-12, MP13-03, V03-08, V15-09

FUCHS, G, MP11-17

FUGARU, I, MP25-14

FUGITA, OE, MP26-07

FUJIMOTO, T, MP12-06

FUJISAWA, M, MP19-22

FUKAMI, N., BSP1-03

FUKAYA, K., BSP1-03

FUKUMA, Y, MP26-10

FUKUNAGA, A, MP17-17

FUKUZUMI, S., BSP1-19

FULLA, J, MP11-19, V09-11, V15-10

FUNAHASHI, Y, MP01-11

FURUKAWA, J, MP19-22

FUSCHI, A., BSP1-14

G

GABOARDI, F, MP02-13, MP15-23, MP26-21, V18-10

GADE, A, MP06-01, MP06-12

GADELMOULA, M, MP07-22

GAHAN, JC, MP06-05, MP09-20

GALLANTE, B, MP05-07, MP05-10, MP22-01, MP23-09, MP23-15, MP30-12, MP30-22

GALLIOLI, A, BSP1-16, MP01-02, MP16-20, MP18-08, MP19-20, V02-08

GALLO, B, MP12-11

GALLO, N, MP12-11

GALLUCCI, M, MP09-05, MP11-20, MP14-19, MP15-15, MP15-18, MP17-20, MP18-19,

MP18-21, V08-11, V14-06

GALLYAMOV, E, MP15-07

GAN, C, MP20-04 
GANAPATHI, H, MP12-19

GANESAN, S, V10-07, V12-04

GANGE, S, MP02-20

GANPULE, A, BSP2-03, MP10-08, MP19-10, MP25-17, MP26-20, V15-05

GAO, Z, MP01-03, MP01-08, MP21-06

GARAYEV, A, V02-09, V03-10, V08-01, V11-08, V14-05

GARBENS, A, MP06-05, MP09-20, MP13-10, MP14-11, V09-07, V13-01

GARCÍA-RIPOLL, J TORRECILLA, BSP1-08, MP16-22, V17-03

GARCÍA, J FRANCISCO SÁNCHEZ, MP21-12

GARCIA, J TORRES, MP23-12

GARCIA, S GRACIA, MP22-21

GARCÍA ROMERO, A, MP21-12

GARG, G, MP29-09

GARISTORISCO, J, V09-08, V09-10, V13-02, V13-04, V13-07, V13-11, V13-12

GARROD, H, MP05-03

GAUHAR, V, MP29-02

GAUSA, L, MP19-20

GAVRILOV, P, MP16-20

GAYA, J, MP01-02, MP16-20

GAZDAR, T, MP30-18

GAZEL, E, MP02-06, MP08-20, V01-07

GEAVLETE, BF, MP25-08, MP25-21

GEAVLETE, PA, MP25-08, MP25-21

GEHRING, T, MP08-01, MP08-13

GENGQING, W, MP15-03, MP15-08, MP15-10, MP15-19, MP25-12, MP27-13, MP28-12

GENTILE, B, MP16-05, V17-08

GEORGE, AK, MP14-16

GEORGESCU, DA, MP25-08, MP25-21

GEORGIOU, R., BSP1-12

GEORGOULEAS, P, MP07-15

GERAGHTY, R, MP14-21, MP21-13, MP24-10, MP29-21

GERAKARIS, A, MP26-03, MP30-03

GERBER, G, MP27-12, MP27-16

GERIAL, EL, Jr., MP13-14

GERMINALE, F, MP08-09

GHALI, F, MP26-11

GHAMARIAN, P, MP21-07

GHANDOUR, R, MP14-11

GHANI, K, MP20-07, MP25-05, MP30-04, MP30-16

GHANI, M, MP29-04

GHAZI, A, V03-11, V06-03

GHAZWANI, Y, MP26-17

GHICULETE, D, MP30-13

GHONIEM, G, MP11-07

GIACOBBE, A, MP08-09, MP14-19, MP17-20

GILBERT, N, MP01-18

GILBERT, Z, MP24-07

GILL, A, MP03-13

GILL, N, MP02-21

GILLING, PJ, MP03-07, MP03-20

GIONA, S, V01-11

GIRAUDO, VI, MP20-01

GIULIANELLI, R, MP16-05, V17-08

GIUSTI, G, MP02-13, MP07-10, MP23-12,

MP25-13, MP26-21, MP29-19, MP30-08, V18-10

GKIALAS, K, MP26-03, MP30-03

GLAVIN, K, MP03-19, V07-05

GLYBOCHKO, P, MP04-14

GMORA, S, MP22-06, MP22-17
GOBLE, M, MP06-18, MP16-13

GODBOLE, H, MP08-16, MP13-08, MP19-05, MP19-18

GOEL, R, BSP2-12

$\mathrm{GOH}, \mathrm{A}, \mathrm{V} 08-03$

GOHNO, S, MP12-15

GOKCE, M, MP07-04, MP23-12

GOLASH, A, MP25-04

GOLIJANIN, B, MP20-18

GOLIJANIN, D, MP20-18

GÓMEZ-SANCHA, F, MP03-18, V07-03

GONÇALVES, C, MP28-22

GONDO, T, MP15-16

GONDRAN-TELLIER, B, MP30-06

GONTERO, P, MP14-19, MP17-20

GONZÁLEZ, J CORTIÑAS, BSP1-08, MP16-22,

V01-12, V17-03

GONZÁLEZ, R. CALVO, BSP1-08

GONZALEZ-SANTANA, CA, V10-09

GONZALEZ, D, MP25-18

GONZALEZ, L LLANES, MP03-18, V07-03

GONZALEZ, M, MP23-12, MP26-07

GONZALEZ, R CORTIÑAS, V03-12, V12-05, V16-06

GOOD, E, MP20-17

GOODMAN, J, V05-05

GOPI, P, V10-07, V12-04

GORDON, K, MP09-21

GORIN, MA, MP14-01

GORLA, J, MP04-17

GOTOH, M, MP01-05, MP01-11, MP12-18

GOVOROV, A, MP09-12

GOWER, B, MP04-18, MP22-04

GOYAL, A, MP02-12, MP22-18

GOYNE, A, MP04-08, MP21-22

GÖZEN, AS, MP01-15, MP06-08, MP06-13, MP06-17, MP11-16, MP15-07

GRADY, R, MP20-08

GRAJALES, V, MP07-19

GRALNEK, DR, MP06-02

GRECO, S, MP11-11

GREEAR, G, MP09-16

GREEN, J, BSP2-11, MP01-06

GREENSPUN, A, MP24-17

GREICO, A, MP24-19

GRIER, D, MP02-20

GRIFFIN, CM, MP06-11

GRIFFIN, S, MP27-07

GRIFFITHS, L, MP20-09

GROEGLER, J, MP04-08, MP21-05, MP21-22, MP24-07

GROSS, A, MP11-15, MP29-03

GRUNERT, R, MP01-14

GU, C, MP20-20

GUAGLIANONE, S, MP09-05, MP11-20, MP14-19, MP15-15,

MP15-18, MP17-20, MP18-19, V08-11, V14-06

GUANGYAN, L, MP23-10

GUARINO, G, MP08-18, MP26-02

GUELFII, RA, V16-04

GUGLIELMETTI, G, MP15-20

GUIGGI, P, MP27-18

GUIRADO, L, MP19-20

GUL, N, MP24-21

GUNASEELAN, V, MP20-07

GUO, P, MP06-04, V14-12

GUOXI, Z, BSP1-15, MP01-09, MP15-03, MP15-08,

MP15-10, MP15-19, MP25-12, MP27-13, MP28-12 
GUPTA, M, MP04-04, MP04-15, MP05-07, MP05-10, MP22-01, MP23-09, MP23-14, MP23-15, MP28-14, MP30-12, MP30-22

GUPTA, N, MP20-22

GUPTA, P, V12-13

GUPTA, R, MP29-09

GURBANI, C, V02-01

GURU, K.A., BSP1-10, BSP1-19, MP01-01, MP01-20, MP06-15, MP12-08, MP15-09, MP15-23, MP20-08, V08-04

GURUNG, PM, V15-01, V15-07

GUTIERREZ, E, MP24-06

GUZEL, O, MP08-20

\section{H}

HA, J, MP18-06

HA, U, MP17-01

HAAS, C, MP02-03, MP24-15

HABUCHI, T, MP12-14, MP15-16, MP18-20, V14-11

HADJIPAVLOU, M, MP03-08

HAGER, SP, MP14-09

HAGERTY, J, MP27-02

HAGHPANAH, A, MP07-13

HAJIHA, M, MP04-08, MP14-06, MP21-05, MP21-22, MP24-07, MP30-11

HAJIRAN, AJ, V06-09

HAJJ, A, MP08-14, MP12-03, MP13-18, V01-04

HAKARIYA, T, MP12-15, V05-12

HALE, J, MP05-15, MP16-19, MP20-15, MP26-08, MP27-03, MP30-01

HALEBLIAN, G, MP10-08

HALGRIMSON, WR, MP17-14, MP17-19

HALL, S, MP01-01

HALL, T, MP25-05, MP30-04, MP30-16

HALSTEAD, NV, MP19-12

HAMASAKI, T, MP03-11

HAMDOON, MA, MP01-17, MP29-10, V05-07

HAMID, A, MP27-09

HAMILTON, BD, MP27-12, MP27-16

HAMMAD, F, MP04-10, MP26-05, MP29-17

HAMPTON, L, MP13-01, MP13-09, V11-05

HAMRI, S BIN, MP26-17

HAN, B, V07-10

HAN, D, MP21-15, MP26-09

HAN, J, MP18-01, V06-04

HAN, W, V09-05, V13-06

HANMIN, C, MP01-09

HANNA, E, MP23-19

HANNA, M, MP20-04

HANSOMWONG, T, MP17-08

HARA, S, MP15-02, V16-02

HARADA, K, MP19-22

HARAKE, N, MP23-19

HARBIAS, A, MP21-02

HARINATHA, S, V11-09

HARRAZ, A, V12-09

HARRIS, F, MP19-05

HARTMAN, C, MP24-13, MP24-14

HASAN, A, MP27-01, MP28-02, MP29-15

HASHIM, H, MP09-10

HASHIMOTO, Y, MP12-20, MP15-14, MP17-13

HASHMI, A, MP23-17, MP30-18

HASSEN, W, V05-11
HATAKEYAMA, S, MP12-20, MP15-14, MP17-13

HATORI, M, MP26-10

HATTORI, K, MP01-05, MP09-06, MP12-02

HATTORI, S, MP15-02, V16-02

HAUSMANN, TJ, MP29-03

HAWTHORN, B, MP03-14

HAYAMI, H, MP15-22

HAYASHI, T, MP03-11, MP05-19

HE, C, MP04-04

HE, Q, BSP2-04

HEALY, KA, MP22-14

HEATH, G, MP11-22

HEBERT, K, MP09-14

HEIDAR, NF ABOU, MP12-03

HEMAL, A, MP02-19, MP09-11, MP15-23, MP18-05,

MP18-18, MP18-22, V09-09

HENDERSON, A, MP09-02

HENDRY, J, MP19-15

HENG, C, MP29-02

HENNIG, M, MP01-18, MP29-06

HENRY, P, MP14-13

HEO, J, V13-06

HERGUEDAS, E. Valsero, BSP1-08

HERNÁNDEZ, J, MP24-06

HERNANDEZ-MENDEZ, E, MP25-10, V12-11

HERNES, E, MP12-22

HIKITA, K, MP13-13

HILL, S, MP21-04

HINATA, N, MP19-22

HINDLEY, R, MP08-01, MP08-13

HINDS, F, MP22-18

HINDUSTANWALA, A, MP06-01, MP06-12

HINTZY, M, MP14-13

HIRAYAMA, T, MP12-09

HIROTA, K, V02-03, V04-01

HIRSH, A, MP02-20

HO, A, MP14-21

HO, D, MP04-22

HO, H, MP03-20, MP06-14, MP29-14

HO, H CHING, MP30-10

HODHOD, A, MP03-22, V07-04

HODOTSUKA, N, MP03-11, MP05-19

HOENIG, D, MP20-09, MP21-14, MP24-14,

MP24-19, MP28-16, V04-08, V18-03

HOFF, J, MP12-22

HOLE, K, MP12-22

HOLLINGSWORTH, JM, MP20-07, MP30-16

HOLMES, R, MP04-18

HONDA, M, MP13-13

HONEY, J, MP30-13

HONG, B, MP19-11

HONG, D., BSP1-11

HONG, S, MP17-01

HONG, Y, BSP1-22, MP04-16, MP07-11, MP07-12, MP28-03

HOOGENES, J, MP06-09, MP17-21, MP22-06, MP22-17

HOPLAND, O, MP12-22

HORCAJADA, A ZAMORA, BSP1-08, MP16-22, V17-03

HORIGUCHI, Y, MP12-17

HORN, T, V11-11

HOROWITZ, A., BSP1-17

HOSHINO, T, MP13-13

HOSSEINI, M, MP07-13

HOU, K, V03-04, V05-02

HSIEH, J., BSP1-20 
HSIEH, T, MP09-16

HSU, F, MP09-09

HSU, J, MP26-19

HU, D, MP29-20

HU, G, V02-13

HU, H, MP04-07, MP10-10, MP28-15

HU, M, MP13-15

HU, W, MP20-05, MP23-18

HU, G, V04-11

HUANG, C, MP13-11, V09-01

HUANG, E, MP13-04

HUANG, H, V18-13

HUANG, J, V02-13, V04-11

HUANG, M, MP22-19, V14-11

HUANG, T, MP13-04, MP23-03, MP23-06, MP25-22

HUANG, W, MP13-04

HUANG, X, MP04-16, MP05-18, MP06-04, MP07-11, MP07-12, MP11-10, MP28-03

HUANG, X., BSP1-22

HUANG, Y, MP13-04, MP24-16

HUBOSKY, SG, MP27-02

HUGHES, S.F., BSP2-02, BSP1-05, MP03-13

HUGHES, T, MP16-06, MP21-13

HUGO, S, V16-05

HULL, B, MP15-09

HUMPHREYS, MR, BSP1-09, MP05-20, MP09-04

HUPE, MC, MP01-18, MP29-06

HUQ, S, MP13-08

HUR, D, MP30-11

HUSSAIN, M, MP18-10, MP23-17, MP30-18

HUSSEIN, AA, BSP1-19, MP01-01, MP01-20, MP06-15, MP12-08, MP15-09, MP20-08, V08-04

HUSSEIN, Y, MP08-21, V01-10

HUYNH, LM, MP01-10, MP01-12, MP14-01, MP17-16

HYAMS, E, MP02-03, MP24-15

I

IBRAHIM, A, BSP2-05, MP08-05, MP08-07

IBRAHIM, I, MP11-18, MP14-07, MP19-15

ICHII, H, MP11-08

ICHINO, M., BSP1-03

IDRIS, F, MP29-22

IEONG, K, MP24-05

IHARA, T, MP13-17

IIJIMA, H, MP12-06

IKEDA, M, MP16-02

ILBEY, Y, MP25-20

ILGI, M, BSP2-10, BSP2-17, MP03-03, MP23-02

ILIAS, D, MP11-05, MP17-04

ILKI, Y, V02-04, V06-02

IMAI, A, MP12-20, MP15-14, MP17-13

IMAMURA, M, MP12-06

INKAYA, A, MP17-09

INMAN, R, MP06-20

INOKUCHI, J, MP11-13

INOUE, K, MP12-01, MP13-20, MP16-04, MP17-17

INOUE, S, MP01-11

INOUE, T, MP12-14, MP18-20, V14-11

IORGA, M, V03-03

IPPOLITO, J, MP19-13

IQBAL, N, MP07-14, MP27-01, MP28-02, MP29-15

IRANI, D, MP07-13

IRIE, A, MP16-02
IRIE, S, MP11-13

ISHIDA, M, V14-04

ISHIDA, R, MP12-18

ISHII, D, MP16-02

ISHIMURA, T, MP19-22

ISKANDER, M, MP05-03

ISMAIL, M, MP12-13, V11-04

ISSA, R, MP15-06

ITO, J, MP12-10

IWAMI, D, V10-03

IWAMOTO, H, MP13-13, MP15-16

IWAMURA, M, MP12-09, MP16-02

IZMAILOV, A, BSP2-21

IZUMI, K, V02-03, V04-01

J

JAAFAR, R, MP08-14

JABEEN, M, MP23-17

JACKSON, B, BSP2-20

JACOBSOHN, K, MP13-01

JADHAV, K, V01-01

JAGTAP, J, MP24-11, V01-01, V10-11

JAIN, S, BSP2-12

JALADANKI, S, MP04-04

JAMBOR, I, MP02-01, MP19-01

JAMES, B, MP02-04, MP20-19

JAMES, G, MP01-20, MP12-08

JAMIL, S, MP29-04

JAMJOOM, R, V15-12

JANG, TL, MP19-21

JANG, W, V13-06

JARVIS, H, MP23-07

JAVED, S, MP05-03

JAYAWARDENA, P, MP22-10

JEFFERIES, M, MP02-04

JEFFERSON, FA, MP11-07, MP11-08,

MP14-01, MP16-03, MP21-07, V02-11

JENSCH, S, MP04-03

JENSEN, J, V06-01

JENSON, C, MP12-05, MP12-11

JEON, H, MP21-15

JEON, S, MP21-15

JEONG, B, MP19-11

JERAM, V, MP09-01

JHANG, S, MP26-19

JI, W, MP01-20

JIAN, Z, MP09-22

JIANG, P, MP11-14, MP20-11

JIANG, Q, MP04-21

JING, Y, V07-10

JING, Z, MP15-09, MP15-23, V08-04

JINGYANG, G, MP10-15, MP25-16

JOA-CABRERA, M, V10-09

JOHNIN, K, V05-10

JOHNSON, BA, MP06-05, MP09-20, MP13-10,

MP14-11, MP22-09, MP22-11, MP22-16,

MP29-01, MP29-16, V09-07, V13-01

JOHNSTON, M, MP08-01, MP08-13

JONES, M, MP16-19, MP18-12

JONES, P, MP24-10, MP27-15

JONGJITAREE, K, MP14-12

JOSEPH, J, V18-04

JOSEPH, JV, V03-11, V06-03, V15-07 
JOUR, I, MP16-10

JUAN, Y, MP23-03, MP25-22

JUN, M, V11-06, V13-05

JUNG, H, MP26-12

JUNG, W, MP18-06, MP29-07

\section{$\mathbf{K}$}

KADER, K, MP09-16

KAGEYAMA, S, V05-10

KAHN, A, MP19-08

KAID, T, V15-12

KAIHO, Y, MP12-10

KAJE, Y, MP07-06

KAKI, A, V15-12

KALATHIA, JA, MP28-13, MP30-02

KALDANY, A, MP02-05, MP19-04

KALE, N, MP08-16, MP13-08, MP19-05, MP19-18

KALLAPPAN, S, V05-06, V05-09, V12-06, V12-10, V12-12, V16-10

KALLIDONIS, P, MP03-06, MP05-16, MP23-13, MP26-03, MP30-03, MP30-08

KAMAL, N, MP07-21

KAMAL, S, MP01-01

KAMAL, WK, MP07-09, MP16-16

KAMPANTAIS, S, MP09-21

KAN, C, V02-10

KANAO, K, MP15-02, V16-02, V17-02

KANASHIRO, A, MP22-21, V04-04

KANAYAMA, A, MP03-17

KANAYAMA, H, MP15-16

KANDA, S, MP12-14, MP18-20, V14-11

KANDEVANI, N YOUSEFZADEH, MP24-08

KANEKAR, S, MP26-04

KANEKO, G, MP15-02, V16-02, V17-02

KANG, J, MP09-08

KANG, M, MP21-15

KANG, S, MP19-11, V13-06

KAOUK, J, MP13-01, MP13-09, MP13-22, MP15-13, MP15-23, V09-08, V09-10, V13-02, V13-04, V13-07, V13-11, V13-12

KAPLAN-MARANS, E, MP05-07, MP11-09, MP11-19, V09-11, V15-10

KAPOOR, A, MP04-04, MP04-15, MP05-10, MP23-09, MP23-14, MP23-15

KAREEM, Z, MP19-10

KARIM, O, V11-04

KARSENTY, G, MP23-20, MP30-06

KASHYAP, R, V06-03

KASRAEIAN, A, MP08-11, MP08-22

KASSOUF, W, BSP2-05

KATIMS, A, MP05-10

KATMAWI-SABBAGH, S, MP03-14

KATO, M, MP01-05, MP01-11, MP12-18

KATZ, M, MP02-15, MP21-04

KAUER, PC, MP04-03

KAUFFMAN, E, MP01-01, MP01-20

KAUSHIK, S, MP06-01, MP06-12

KAVIA, R, MP03-09

KAVOUSSI, LR, MP14-01

KAWADA, K, MP12-15

KAWAKITA, M, MP12-01, MP13-20, MP15-16, MP16-04, MP17-17

KAWANISHI, Y, V02-03, V04-01
KAWASAKI, H, V14-04

KAWAUCHI, A, V05-10

KAYA, E, MP02-06, V01-07

KAYES, O, MP03-09

KAZANTIS, G, MP10-02

KAZIHINUROV, R, BSP2-21

KAZMI, Z, MP30-20

KEDDIS, MT, MP05-20

KEEHN, A, MP09-19, MP17-10

KEIRANDISH, P, MP16-19, MP20-15, MP26-08,

MP27-03, MP30-01

KELLER, M, MP22-12

KELLY, P, MP05-03

KESHAVAMURTHY, M, V11-09

KHADGI, S, MP28-10

KHAIRULLINA, R., BSP1-21

KHALAF, MI Al, V06-05

KHALIQUE, A, MP23-17

KHAN, A, MP02-16, MP02-20

KHAN, F, MP03-13

KHAN, M, MP06-11, MP06-13, MP06-17, MP06-18,

MP11-16, MP16-14, MP20-17

KHAN, N, MP25-02, MP28-01

KHAN, N., BSP1-01

KHAN, R SOHAIL, MP20-21

KHAN, S, MP15-06

KHATIB, F el, MP01-12, MP17-16

KHAULI, R, MP13-18

KHEIRANDISH, P, MP20-10

KIKUCHI, H, V10-03

KILAVUZOĞLU, AE, MP16-17

KIM, AI, MP28-05

KIM, B, MP18-06

KIM, C, MP18-06

KIM, D, BSP2-15, MP18-02, MP18-13, MP18-17,

V09-05, V13-10, V15-04

KIM, E, MP06-22, MP20-20

KIM, E., BSP1-20

KIM, H, MP18-07, MP23-05

KIM, IY, MP19-21

KIM, J, MP01-13, MP09-17, MP12-04, MP20-20

KIM, K, MP18-01, V06-04

KIM, S, BSP1-11, MP17-01, MP19-11

KIM, T, MP20-07

KIM, Y, MP18-01, MP26-12, MP27-11, V06-04

KIM, M, MP09-08

KIMURA, G, MP05-19

KIMURA, Y, MP13-13

KINOSHITA, H, MP21-18

KIRA, S, MP13-17

KIRECCI, S, BSP2-10, BSP2-17, MP03-03, MP23-02

KIREMIT, MC, V08-09

KISLYAKOV, D, MP01-04

KK, Y, MP08-08, MP29-13, V01-06, V04-03

KLEINKLAUSS, F, MP21-21

KLETT, D, MP27-12, MP27-16

KLIMOV, R, MP10-17, MP30-05

KNAUER, C, MP14-22

KNIGHT, J, MP04-18

KNUDSEN, BE, MP10-05, MP10-09, MP10-16, MP11-02,

MP11-06, MP21-19

KO, Y, MP26-12

KOAUK, J, MP11-20, MP18-21

KOBAYASHI, H, MP12-18, V14-04 
KOBAYASHI, M, MP25-01

$\mathrm{KOCH}, \mathrm{B}, \mathrm{MP} 11-22$

KOGA, S, MP12-17

$\mathrm{KOH}, \mathrm{D}, \mathrm{V} 13-06$

KOHADA, Y, MP12-10

KOIE, T, MP15-16

KOIKE, H, MP03-17

KOJIMA, S, V03-04, V05-02

KOKUBUN, H, MP17-17

KOLDEWIJN, E, MP19-09

KOMARAVOLU, SS, BSP2-15, MP18-02, MP18-13, MP18-17, V13-10, V15-04

KOMATSU, K, MP09-06

KOMMU, SS, MP06-19, MP14-02, MP14-04, MP14-08, MP15-06, MP16-19, MP17-03, MP17-07, MP18-12, MP19-03, MP20-10, MP20-15, MP22-05, MP26-08, MP27-03, MP30-01, V11-12

KONAY, A, V05-08

KONDJIN-SMITH, M, MP20-04

KONDO, Y, MP03-11, MP05-19

KONGCHAROENSOMBAT, W, MP12-21, MP19-02, V03-09

KONNAI, Y, V14-04

KOO, K, MP10-11, MP10-13, MP20-22, MP22-02

KOROLEV, D, MP04-14, MP10-17, MP30-05

KORPRAPHONG, P, MP14-12

KOSEOGLU, E, V08-09

KOSTER, H, BSP2-15, MP14-14, MP18-02, MP18-13, MP18-17

KOSUGI, M, V14-04

KOTB, A, MP03-22

KOTHARI, PD, BSP2-15, MP18-02, MP18-13,

MP18-17, MP18-18, V13-10, V15-04

KOTSIRIS, D, MP03-06

KOTT, O, MP20-18, MP22-19, MP26-01

KOWN, S, MP30-11

KOZEL, Z, MP20-09

KOZLER, CJ, MP06-02

KRAMBECK, A, MP04-12, MP05-13, MP09-13, MP09-18, MP10-04, MP23-07, MP28-07, MP30-15, V01-03, V01-05, V17-06

KRAMER, F, MP25-18

KRAMER, MW, MP01-18, MP22-20, MP29-06

KRAUSE, A, MP14-06

KRETOV, M, V06-08

KRIPLANI, J, MP13-21

KRISHNAN, J, MP06-20

KRISHNAN, R, MP16-19, MP20-10, MP20-15, MP26-08, MP27-03, MP30-01

KU, J, MP19-11

KUBOTA, M, MP12-01, MP13-20, MP16-04, MP17-17

KUCHERIA, R, MP02-12, MP22-18

KUCUK, E, MP17-09

KUDO, T, MP12-10

KUETTEL, M, MP01-01

KULKARNI, A, BSP2-22

KUM, F, MP16-14

KUMAR, A, V05-04, V12-02, V16-03

KUMAR, P, MP14-19, V11-09

KUMAR, R., BSP1-19

KUMAR, S, V05-04, V12-02, V16-03

KUNLIN, X, MP01-09

KUO, J, MP13-04

KUO, W, MP02-17, MP18-14, MP26-15
KUO, Y, MP14-14, MP18-17

KURAL, A, MP16-17, MP18-15

KURTI, M, MP08-09

KUSAKA, M., BSP1-03

KUSTERS, A, MP07-03

KUSUMA, V, MP15-06

KUTLIYAROV, L.M., BSP1-21

KWON, O, MP01-22, MP02-10

KWON, T, MP13-07

KWUN, H, MP04-15, MP05-10, MP23-09, MP23-14, MP23-15

KYONO, Y, MP09-06

KYRIAZIS, I, MP03-06

$\mathbf{L}$

L'ESPÉRANCE, S, MP25-07

LABBAN, MS, MP12-03

LABBATE, C, MP20-02

LADDHA, A, MP11-21, V09-04

LAFLAMME, N, MP25-14

LAGACÉ, S, MP25-14

LAI, K, V02-10

LAL, M, MP23-17

LAM, L, V02-10

LAMOUSHI, A, MP13-05

LANA, BN, MP30-19

LANDIN, M, MP16-14

LANDMAN, J, MP05-14, MP10-20, MP11-07, MP11-08, MP11-14, MP14-01, MP16-03, MP21-07, V02-11

LANDMESSER, J, MP22-20

LANGE, D, MP04-20

LANGENHUIJSEN, H, MP07-03

LANGENHUIJSEN, J, MP15-07

LAPITAN, M, MP07-05, MP28-06

LARCHER, A, MP18-21

LARGE, T, MP04-12, MP05-13, MP09-13, MP09-18, MP10-04, MP23-07, MP28-07, MP30-15, V01-03, V01-05, V17-06

LARSON, E, MP06-05

LARSON, T, MP06-10

LATIF, E, MP05-15, MP06-19, MP14-02, MP14-04, MP14-08, MP15-06, MP16-19, MP17-03, MP18-12, MP19-03, MP27-03, V11-12

LATIMER, T, MP09-01

LATURNUS, JM, MP29-06

LAU, C, MP13-01

LAU, W, MP06-14

LAZAROWICZ, H, MP05-03

LEÃO, R, MP24-12, MP28-22

LE, BV, MP06-02

LEAVITT, D, MP20-07

LECHEVALLIER, E, MP23-20, MP30-06

LEE, D, MP18-07, MP23-05, MP29-07

LEE, DI, MP01-13, MP09-17, MP12-04, MP19-07, MP20-20, V11-01

LEE, H, MP01-22, MP02-10, V09-05

LEE, J, MP17-01, MP29-11

LEE, J., BSP1-06

LEE, JY, MP30-13

LEE, K, MP23-05

LEE, M, MP09-19, V06-11

LEE, S, MP18-07, MP23-05 
LEE, T, MP11-07

LEE, Y, MP02-11, V13-06

LEE, YJ, MP17-14

LEE, Z, BSP2-15, MP09-19, MP17-10, V06-11, V11-10

LEENANUPAN, C, MP12-21, V03-09

LEEWANSANGTONG, S, MP02-22, MP17-08

LEFFLER, TG, MP06-02

LEITE, K, MP11-05

LEKAREV, V, MP10-17, MP30-05

LESTER, JF, MP14-20

LETENDRE, J, MP21-21

LEVINE, JP, V11-06

LEWIS, BJ, MP06-02

LEWIS, SC, MP02-01, MP14-16, MP14-22

LI, C, MP01-03, MP01-08, MP21-06, MP23-03, MP25-22

LI, G, MP11-07, MP22-14

LI, H, BSP2-04, BSP2-18, MP01-21, MP04-21, MP09-22, MP19-14

LI, J, MP04-01, MP07-16, MP20-05, MP22-03, MP22-19, MP23-08, MP23-18

LI, M, MP01-03, MP01-08, MP21-06

LI, Q, MP01-01

LI, W, V09-01

LI, Y, MP04-21, MP13-03, V15-09

LI, Z, MP16-18

LIAO, B, MP19-14

LIATSIKOS, E, MP01-15, MP03-06, MP05-16, MP06-08, MP10-03, MP23-13, MP26-03, MP30-03, MP30-08

LICHTBROUN, BJ, V10-08

LIM, E, MP06-14

LIM, S, MP10-11

LIM, Y, MP03-20

LIMA, A, MP21-13

LIMA, E, MP01-19, MP06-07, MP16-08, MP24-12, MP24-18, MP28-22, V17-10

LIMFUECO, L, MP05-14, MP10-20, MP11-08, MP21-07

LIN, A, MP13-04

LIN, C, MP13-04

LIN, G, MP30-14

LIN, J, MP02-17, MP18-14, MP26-15

LIN, K, V15-03

LIN, T, MP01-07, MP13-04, MP26-19, V14-02, V18-13

LIN, W, MP26-19

LIN, X, MP05-11, MP23-10

LIN, Y, V14-02

LIPKIN, M, MP04-22, MP21-20

LITVIN, IE, V16-04

LIU, C, V18-13

LIU, H, MP06-04

LIU, L, MP19-14

LIU, S, MP22-19

LIU, W, BSP2-09, V13-05

LIU, X, MP13-03, V15-09

LIU, Y, MP04-21

LIU, Z, MP01-21

LLOYD, GL, MP19-12

LOBO, N, MP14-04, MP14-08, MP14-15, MP17-03, MP17-07, MP18-12

LOCKYER, R, MP14-07

LOMBARDO, R, MP16-05, V17-08

LONE, Z, MP01-01, MP20-08

LOOCK, P, V03-01, V16-11

LÓPEZ, JM, MP14-10, V10-06, V18-02, V18-11

LOTAN, Y, MP22-09, MP22-11, MP22-16
LOVALLO, G, BSP2-15, MP10-21, MP11-01, MP18-02,

MP18-13, MP18-17, V03-03, V08-10, V09-12, V13-09

LOW, W, V04-07

LOW, Z, MP05-09

LOZADA, E, MP07-18, MP22-22

LU, S, MP13-04, MP16-03, MP21-07

LU, Y, MP04-07, MP10-10, MP10-18, MP23-04, MP28-15

LUBBAD, L, MP04-10

LUCIANO', R, MP26-21

LUNA, A, MP26-07

LUO, D, BSP2-04

LUO, H, MP02-08

LUO, S, MP29-20

LUPERTO, E, V16-07

LUQUE, P, MP14-10, V10-06, V18-02, V18-11

LYBBERT, DT, V18-01, V18-05

LYNCH, E, MP24-19, MP28-16

LYU, J, MP06-04

M

MA, K, MP07-11

MA, L, MP19-14

MAATMAN, T, MP15-23

MACCONAGHY, B, MP25-05

MACKINLAY, J, MP06-19

MADAAN, S, MP01-16, MP01-17, V05-07

MADI, R, MP15-21, MP17-11

MAESTRO, MA, MP30-08

MAGALHAES, G, MP11-05, MP17-04, MP17-05

MAGHSOUDI, R, MP24-08

MAHER, E, MP22-18

MAHMALJ, W, MP03-05, MP21-02, MP29-05,

V04-12, V07-01, V07-02

MAHMOOD, SN, MP07-02, MP29-18

MAHMUD, SM, MP25-11

MAIA, R, MP11-05, MP17-04, MP17-05

MAIENKAR, H, MP26-04

MAILHAC, A, MP08-14, MP12-03, MP13-18

MAINEZ, J ANTONIO, MP21-12

MAJIMA, T, MP12-18

MAKANJUOLA, J, MP06-13, MP11-16

MAKITA, N, MP12-01, MP13-20, MP16-04, MP17-17

MAKSIMOVA, S, BSP2-08

MALALLAH, B, BSP2-06, BSP2-13

MALDONADO, J, MP04-08, MP21-05, MP24-07, MP30-11

MALHOTRA, A, MP12-04

MALOSSINI, G, MP17-20

MANGIATORDI, A, MP28-14, V18-07

MANICKAM, R, V05-06, V05-09, V12-06, V12-10, V12-12, V16-10

MANSI, H, MP30-21

MANZO, B, MP04-19, MP07-04, MP07-18, MP22-22, MP23-12

MAO, R, MP11-07

MARCHANT, F, MP26-07

MARCHIONI, M, MP02-13

MARENCO, J., BSP1-07

MARGHAWAL, D, MP13-02, V05-03, V10-02

MARIEN, T, MP22-09, MP22-11, MP22-16

MARKO, A., BSP1-19

MARROIG, B, MP30-19

MARSDEN, T, MP24-22

MARSH, H, MP29-10 
MARTÍN, S MARTÍN, BSP1-08, MP16-22, V01-12, V03-12, V12-05, V16-06, V17-03

MARTÍNEZ, D. RIVERO, BSP1-08

MARTIN, A, MP03-19, MP08-12, MP08-15

MARTIN, M ARRABAL, MP21-12

MARTINEZ-ARROYO, C, MP07-20, MP25-10, V12-11

MARTINEZ, M, MP16-20

MARTINEZ, OE, MP12-07, MP15-12, MP19-16

MARTINI, A, BSP1-17, MP02-01, MP19-01, MP28-14

MARTOCCIA, A., BSP1-14

MARTOS, R, V10-06

MARTOV, A, V02-06, V17-04, V17-09

MARUCCIA, S, BSP2-16, MP03-04

MASSIE, P, MP05-05

MASTROIANNI, R, MP09-05, MP11-20, MP14-19, MP15-15, MP15-18, MP17-20, MP18-19,

MP18-21, V08-11, V14-06

MASUDA, H, V03-04, V05-02

MATHEW, S, MP25-04

MATLAGA, B, MP10-11, MP10-13, MP20-22, MP22-02

MATSUDA, D, MP16-02

MATSUDA, T, MP12-15, MP21-09, MP21-18

MATSUI, H, MP03-17

MATSUKAWA, Y, MP12-18

MATSUMOTO, ED, MP06-09, MP17-21, MP22-06, MP22-17

MATSUMOTO, K, MP12-09, MP15-16, MP16-02

MATSUMOTO, R, V10-03

MATSUO, T, MP12-15

MATSUOKA, T, MP12-01, MP13-20

MATSUZAKI, J, MP21-09

MATTHEW, S, MP18-12

MATULEWICZ, R, V08-03

MAUGERI, O, MP07-04

MAVILLA, L, MP16-05, V17-08

MAXWELL, AD, MP25-05

MAY, N, MP14-09

MAY, P, MP15-23, MP20-08

MAYO, A RUANO, BSP1-08, MP16-22, V17-03

MAZZUCCHI, E, MP07-04

MCADAMS, S, MP18-09

MCCAHY, P, MP05-04

MCILHENNY, C, MP06-11

MCMARTIN, C, MP25-07

MEARINI, E, MP27-18

MEDEIROS, RM, MP30-19

MEENAKSHI-SUNDARAM, B, V06-01

MEHDI, S, MP08-17, MP15-04

MEHMOOD, S, MP02-02

MEHRAZIN, R, MP02-19, MP18-05, V17-05

MEHROTRA, S, MP08-17, MP15-04

MEHTA, S, MP24-19

MEIJER, R, MP19-09

MEILIKA, K, MP02-19, MP18-05, MP18-11, V01-08,

V08-12, V09-03, V11-07, V14-07

MEJÍA-ROSARIO, JP, V10-09

MEKHAIL, M, MP28-16

MEKHAIMAR, A, MP16-13

MELHEM, M, MP30-21

MELNYK, R, V03-11, V06-03

MELO, P, MP07-04

MENDES, A LOPES, MP16-05, V17-08

MENDOZA, KA, MP26-16, MP28-04

MENEAN, M, MP05-06

MENEGHINI, A, MP08-18
MENESES, J, MP05-01

MENG, X, V13-05

MENON, M, MP15-23

MERCADÉ, A, V02-08

MERHEB, S, MP03-21, MP21-04

MERIA, P, MP16-10, MP25-09

MERSEBURGER, AS, MP01-18, MP22-20, MP29-06

MESKAWI, M, MP08-03

MEYER, A, MP20-22

MIAH, S, MP20-04

MICALI, S, MP04-13, MP08-18, MP16-11, MP26-02, MP27-17

MICHEL, F, MP23-20

MIKAMI, H, MP03-11

MIKAMI, J, MP12-10

MIKAMI, K, V03-04, V05-02

MIKHAIL, D, MP02-14, MP20-09, MP21-14, MP24-19, MP28-16, V18-03

MIKHAYLIKOV, T, MP15-07

MILANDRI, R, MP04-13, MP08-18, MP16-11, MP26-02, MP27-17

MILESI, R, MP08-21, V01-10

MILLER, BL, MP06-02

MILLER, D, MP07-19

MILLER, J, MP24-05

MILLER, N, MP22-09, MP22-11, MP22-16

MINERVINI, A, MP13-01, MP13-09, MP18-21

MINISOLA, F, MP09-05, MP11-20, MP15-15,

MP15-18, MP18-19, V08-11, V14-06

MIRABILE, G, MP16-05, V17-08

MIRDHA, K, MP27-19

MISHRA, DK, MP28-21

MISRAI, V, MP03-10

MISSERT, J., BSP1-19

MISTRY, P, MP02-14, MP20-03

MISTRY, S, MP24-11, V01-01, V10-11

MISURACA, L, MP09-05, MP15-18, V08-11

MITRE, A, MP13-22, V12-08

MITSUI, T, MP13-17

MITSUNARI, K, MP12-15

MIYAO, T, MP26-10

MIYATA, Y, MP12-15, V05-12

MIYAZAKI, J, MP21-09

MIYAZAKI, Y, V14-04

MIYAZAWA, Y, MP03-17, MP26-10

MIYOSHI, K, MP12-16

MOEHR, S, V11-11

MOGHISI, R, MP04-08, MP14-06, MP21-22

MOGHUL, M, MP23-16, MP30-07

MOHAMMAD-SALEHI, S, MP13-12

MOHAMMAD, S., BSP1-01

MOHAMMED, BO, MP07-02

MOHAPATRA, A, MP07-19

MOHLER, J, MP01-20

MOHSIN, R, MP18-10

MOLAWI, A, MP17-21

MOLINA, WR, MP10-05, MP10-09, MP10-16, MP11-02,

MP11-06, MP21-19

MOLINARI, F, MP03-04

MONDA, S, MP06-22

MONGA, M, MP03-01, MP07-04

MONTERO, J, MP24-06

MONTORSI, F, MP05-06, MP18-21, MP29-19

MOOCHHALA, SH, MP22-18 
MOON, H, MP17-01

MOON, K, MP09-08, MP13-07, MP26-12

MOORE, C, MP17-21

MOORE, J, V06-10

MOORE, SL, BSP2-02, MP03-13

MORA, E Rijo, MP03-10

MORAIS, N, MP01-19, MP06-07, MP24-12, MP24-18, MP28-22

MORALES-MONTOR, G, MP07-20, MP25-10, V12-11

MOREIRA, C, MP15-20

MORGAN, M, MP09-01

MORING, N, MP24-17

MORINI, E, MP27-17

MORIYASU, E, MP13-13

MORIZANE, S, MP13-13, MP15-16

MORLEY, CE, V06-09

MORRISON, I, MP14-04, MP14-08, MP14-15

MORROW, J, MP08-04

MOSCHONAS, D, MP15-06

MOSTAFID, H, MP15-06

MOTA, P, MP01-19, MP06-07, MP24-12, MP24-18, MP28-22

MOTTA, G, BSP2-16, MP03-04

MOTTRIE, A, MP13-01, MP13-09, MP18-21

MOTZER, J, MP03-02

MOUAD, N, MP27-08

MOUKARZEL, M, MP23-19

MOURÃO, T, V03-06

MOZER, P, MP14-19

MUELLER-LEONHARD, C, MP20-18

MUELLER, T, MP02-20

MUGHAL, A, MP28-02

MUGHAL, M, MP27-01, MP29-15

MUGIYA, S, MP21-09

MUKHERJEE, S, MP01-16, MP21-10, MP21-17

MÜLCHI, C, MP25-18

MULTESCU, R, MP25-21

MUNEER, A, MP16-13

MUNSHI, F, V08-02

MUNVER, R, BSP2-15, MP10-21, MP11-01, MP18-02, MP18-13, MP18-17, V03-05, V13-08, V14-08

MURAI, S, V10-03

MURAKAMI, Y, MP12-09

MURALI-KRISHNAN, S, MP06-20

MURATA, S, MP12-01, MP13-20, MP16-04, MP17-17

MURO, Y, MP12-06

MURTAZA, M, MP23-11

MUSQUERA, M, MP18-08, V18-11

MUTO, G, MP08-09, MP14-19, MP17-20

MUTO, Y, V14-11

MYLARAPPA, P, BSP2-22

\section{$\mathbf{N}$}

NA, J, V09-05, V13-06

NACHIMUTHU, S, V05-06, V05-09, V12-06, V12-10, V12-12, V16-10

NADKARNI, G, MP04-04

NAEEM, S, MP29-04

NAGRA, A, MP15-09

NAHAS, W, MP13-22, MP15-20

NAIK, M, MP14-14

NAIR, R, MP15-06

NAIR, S, BSP1-17, MP19-01

NAITO, S, MP12-16
NAKADA, SY, MP05-14, MP06-02, MP22-09, MP22-11, MP22-16, MP27-12, MP27-16

NAKAGAWA, K, MP11-13

NAKAGAWA, M, MP15-22

NAKAGOMI, H, MP13-17

NAKAJIMA, S, MP18-20

NAKAMURA, C, MP12-06

NAKANISHI, H, MP12-15

NAKANO, Y, MP19-22

NAKAYAMA, H, MP03-17

NALAGATLA, S, MP07-08, MP11-18, MP14-07, MP19-15, MP26-18

NAMDARIAN, B, MP03-08

NANE, I, MP24-09

NARA, T, MP12-14, MP18-20, V14-11

NARIMOTO, K, MP09-06

NARITA, M, V05-10

NARITA, S, MP12-14, MP18-20, V14-11

NASRELDIN, M, MP19-20

NAVARATNAM, A, BSP1-09, MP05-20, MP09-04, MP18-09

NAVARRETE, FE, MP26-16

NAYA, Y, V03-04, V05-02

NAZIM, SM, MP25-02, MP28-01, MP29-04

NAZIR, M, MP28-02

NAZZANI, S, BSP2-16, MP03-04

NEDAS, T, MP08-01, MP08-13

NEELAGAR, B, V11-09

NEGRE, T, MP23-20

NETSCH, C, MP11-15, MP29-03

NEVO, A, BSP1-09, MP05-20, MP09-04

NG, A, MP24-14

NG, KL, V01-11

NGUYEN, D, MP08-11

NICHOLAS, B, MP22-19

NIEWAD, E, MP30-08

NING, C, MP23-08

NISHIKAWA, R, MP13-13

NISHIKIMI, T, MP12-18

NISHIMATSU, H, MP11-13

NISHIMOTO, K, V17-02

NIU, Y, V12-03

NOBBS, M, V17-01

NOEL, J, MP15-06

NOMURA, M, MP03-17

NOMURA, S, MP03-11

NOTT, L, MP04-11

NOTTINGHAM, CU, MP04-12, MP05-13, MP09-13,

MP09-18, MP10-04, MP23-07, MP28-07, MP30-15,

V01-03, V01-05

NOURELDIN, YA, MP21-11

NOURISSAT, A, MP25-07

NTASIOTIS, P, MP03-06, MP23-13, MP26-03, MP30-03

NUALYONG, C, MP02-22, MP14-12

NUMAKURA, K, MP12-14, MP18-20, V14-11

NÚÑEZ, M BEDATE, BSP1-08, MP16-22, V17-03

NUNEZ-NATERAS, R, MP18-09

\section{O}

O'LEARY, M, MP10-20, MP11-08

OBAYASHI, K, MP03-11

OBEK, C, MP16-17, MP18-15

OBI-NJOKU, O, BSP2-02, MP03-13

OCHOA, S COSTAS, MP21-12 
OCHS, S, MP02-20

ODERDA, M, MP14-19, MP17-20

OEZSOY, M, MP05-16, MP30-08

OGATA, A, MP12-17

$\mathrm{OH}, \mathrm{C}, \mathrm{MP02-11}$

OH, T, MP29-11

OHASHI, T, MP12-18

OHBA, K, MP12-15

OHTSUKA, K, V03-04, V05-02

OHWAKI, K, MP09-06, MP12-02

OHYAMA, C, MP12-20, MP15-14, MP17-13

OKA, D, MP03-17

OKABE, T, V17-02

OKEKE, Z, MP02-14, MP20-09, MP21-14, MP24-14, MP24-19, MP28-16, V04-08, V18-03

OKHAWERE, KE, MP02-05, MP02-19, MP18-05, MP18-11, MP18-13, MP18-18, V01-08, V08-12, V09-03, V11-07, V14-07

OKHUNOV, Z, V02-11, MP05-14, MP11-07, MP14-01, MP16-03

OKSAY, T, MP08-20

OKUNO, N, MP16-02

OLAOMI, T, MP22-05

OLIVEIRA, CA, MP16-08, V17-10

OLIVEIRA, R, V03-06

OLSEN, M, MP22-12

OLUWOLE-OJO, A, MP03-08

OMAR, M, MP03-01, MP21-11, V07-08

OMIDELE, O, MP18-03

OMOROGBE, AR, MP23-14, MP23-15

ONAL, B, MP25-06, V02-04, V06-02, V08-05, V08-06, V14-03

ONDARZA, C, MP25-18

ONOL, F, MP06-10, MP12-11

ORDON, M, MP30-13

ORTA, C D', MP02-13

ORTEGA-GONZALEZ, M, MP25-10

ORTEGA, L. PESQUERA, BSP1-08

ORTIZ, C TORRECILLA, MP21-12

OSANN, K, MP05-14

OSAWA, S, MP05-19

OSAWA, T, V10-03

OSMAN, AZ, V04-09

OSMAN, B, MP05-15, MP06-19, MP14-02, MP14-04, MP16-19, MP17-03, MP17-07, MP18-12, MP20-10, MP26-08, MP27-03

OSMAN, MM, MP01-12

OSMAN, N, MP06-20

OSORIO, MA, MP26-16

OTAKE, N, MP26-10

OTSU, A, MP03-17

OTTOSSON, F, MP12-22

OU, C, V14-02, V15-03

ÖNER, A, MP02-06

ÖZDEMIR, H, MP02-06

ÖZMAN, O, MP25-06

OYAMA, M, MP15-02, V16-02, V17-02

OZCAN, F, MP24-09

OZGOK, Y, MP15-07

OZGUR, G, MP28-11

OZIMEK, T, MP01-18, MP22-20, MP29-06

oZISIK, o, MP16-17

OZKAN, O, MP24-21

OZONE, F, V03-01, V16-11
$\mathbf{P}$

PÉREZ-FUENTES, D, MP21-12

PÉREZ, F LARA, BSP1-08, MP16-22, V03-12, V12-05, V16-06, V17-03

PACE, KT, MP30-13

PACHARATAKUL, S, MP12-21, V03-09

PACHECO-GAHBLER, C, MP07-20, MP25-10, V12-11

PACKER, MG, V06-11

PADAYACHEE, W, BSP2-19

PAGE, T, MP03-09

PAGONIS, K, MP03-06, MP23-13, MP30-03

PAHUJA, A, MP08-04

PAI, A, MP24-22

PAIDAKULA, R, V10-07, V12-04

PAIN, J, MP19-03

PAIS, VM, MP05-14

PAK, J, MP02-03, MP24-15

PAK, R, MP17-02, MP17-06, MP26-14, V06-10

PAKMANESH, H, MP13-12

PALANIAPPAN, S, BSP2-03

PALESE, M, MP11-09, MP11-19, MP18-03, MP20-14, V09-11, V15-10

PALLÍN, M PINA, V17-03

PALLAGANI, L, V12-13

PALOU, J, BSP1-16, MP01-02, MP15-23, MP16-20, V02-08

PALOU REDORTA, J, MP06-11, MP06-13, MP06-17, MP11-16, MP22-21

PAN, H, MP05-04

PANDEY, PK, MP23-22

PANDEY, R., BSP1-10, BSP1-19

PANDEY, S, MP08-17, MP15-04

PANDYA, SS, MP19-21

PANTHIER, F, MP04-06, MP04-09, MP05-22, MP29-12

PAPADOPOULOS, G, MP05-15, MP16-19, MP20-10, MP20-15, MP27-03

PAPAGIANNOPOULOS, D, MP26-11

PAPALIA, R, MP17-20, V16-07

PARADIS, A, MP18-16, MP22-12

PARANJPE, I, MP04-04

PARANJPE, M, MP04-04

PARDESHI, A, MP07-06

PAREEK, G, MP20-18, MP26-01

PARIKH, AK, V18-09

PARIKH, K, MP17-02, MP17-06, MP19-08, MP26-14, V06-10, V07-07

PARIKH, KP, V18-09

PARK, J, MP09-08

PARK, J., BSP1-11

PARK, S, MP13-07, MP20-02, MP29-11

PARK, W., BSP1-11

PARK, Y, MP17-01

PARK, C, MP18-06

PARKER, A, MP22-12, V14-01

PARKHOMENKO, E, MP06-03, MP11-08

PASSEROTTI, C, MP11-05, MP17-04, MP17-05

PASSOS, P, MP01-19, MP06-07, MP24-12, MP24-18, MP28-22

PASTORE, A.A., BSP1-14

PATE, W, V01-09

PATEL, A, MP11-17, V02-07, V14-09

PATEL, A., BSP1-18

PATEL, B, MP08-06

PATEL, H, MP20-22 
PATEL, HR, MP15-06

PATEL, HV, V10-08

PATEL, K, MP09-17

PATEL, M, MP02-20

PATEL, NH, MP18-17, V03-03, V09-12

PATEL, R, MP19-21

PATEL, RM, MP05-14, MP10-20, MP11-07, MP11-08, MP11-14, MP14-01, MP16-03, MP21-07, V02-11

PATEL, V, MP01-13, MP06-10, MP12-05, MP12-11, MP12-19, MP17-12, V03-02, V03-07

PATHAK, R, MP09-11, MP18-22, V09-09

PATIL, A, MP25-17

PATIL, K, MP15-06

PATKI, P, MP30-07

PATNI, LG, MP06-01, MP06-12

PATTNAIK, DP, MP11-04, V05-01

PATTNAIK, MP, MP10-19, MP21-16, MP24-03, MP27-20

PATTNAIK, P., MP10-12

PATTNAIK, PK, MP10-19, MP11-04, MP21-16, MP24-03, MP27-20, V05-01

PATTNAIK, SK, MP10-19, MP11-04, MP21-16, MP24-03, MP27-20, V05-01

PATWA, S, MP21-14

PAUCHARD, F, MP25-18, V18-12

PAULMICHL, AA, V16-04

PAULUCCI, D, MP18-18

PAVLAKIS, P, MP15-06

PAVLOV, V, BSP1-21, BSP2-08, BSP2-21, MP23-01, MP28-18, V08-07, V08-08

PAVLOVA, I, BSP1-02, V17-05

PEABODY, J, MP15-23

PEARCE, A, V15-08

PEARCE, D, MP01-17

PEARLE, M, MP22-09, MP22-11, MP22-16, MP29-01, MP29-16

PEDROSA, I, MP14-11

PEDROSA, JP, MP30-19

PELTIER, A, MP14-19, MP17-20

PENEV, BA, MP09-02

PENEVA, E, MP09-02

PENNISTON, KL, MP05-14

PERALTA-BERNAL, I, V10-09

PERDIGÃO, DC, MP30-19

PERDONÀ, S, MP13-01, MP13-09

PEREIRA, P, MP24-12

PEREZ-OROZCO, A, MP21-14

PERI, L, V18-11

PERLIN, D, V06-08, V16-08

PERRY, M, MP15-06

PERSAD, RA, MP19-03

PETRELLI, F, MP08-21

PETRIDES, N, MP17-03, MP17-07

PETRISOR, D, MP10-11

PHAM, B, V14-01

PHAN, Y, MP03-05, MP29-05, V04-12, V07-01, V07-02, V11-04

PHANG, A SIM SOON, V10-04

PHILLIP, D, MP19-01

PICHARDO-GARCIA, M, V10-09

PICKERSGILL, NA, MP16-12

PICKHARDT, MW, V01-05

PIECHAUD, T, MP14-19, MP17-20
PIERORAZIO, P, MP14-01, MP20-22

PIETRO, C Di, MP04-13, MP27-17

PIETROPAOLO, A, MP05-16, MP05-21, MP10-03, MP14-21, MP16-06, MP21-13, MP24-10, MP27-07, MP27-15, MP29-14, MP29-21, MP30-08, MP30-10, MP30-17, V01-02, V04-10

PIMENTA, R, MP11-05

PINDORIA, N, MP02-12

PINI, G, MP01-15, MP03-12, MP06-08, MP07-10, MP09-12, MP14-17, MP21-03

PIRANVISEH, K, MP20-11

PITA, H RÍOS, MP08-19, MP14-05

PLANTE, M, MP01-14

POLO, M A. ARRABAL, MP21-12

POLOSKEY, T, V02-07, V14-09

POLOTTI, C, V08-02

POOLERI, GK, MP11-21, V09-04

POORE, W, MP22-04

POPANES, C, MP21-10, MP21-17

POPERT, R, MP03-08

POPIOLEK, M, MP07-15

PORPIGLIA, F, MP13-01, MP13-09, MP18-21

PORTER, J, MP02-19, MP13-01, MP13-09, MP18-05, MP18-18

POTHIYEDATH, H, MP03-16, MP11-12, V02-12, V07-06, V07-11, V07-12, V10-05

PRABHAKAR, S, MP08-08, MP29-13, V01-06, V04-03

PRADEEP, K, MP17-20

PRASAD, V, V05-04, V12-02, V16-03

PRASHANT, Y, V05-04, V12-02, V16-03

PRATA, F, V16-07

PRATIHAR, SK, V16-12

PRATTLEY, S, MP14-07, MP30-10

PREMINGER, G, MP04-22, MP10-08, MP21-20

PRETE, C Del, MP04-13, MP16-11, MP26-02

PRINCIPE, P, MP18-04

PROIETTI, S, MP02-13, MP07-10, MP21-03, MP23-12,

MP25-13, MP26-21, MP29-19, MP30-08, V18-10

PROMPONAS, I, MP01-16

PROSKURA, A, MP01-04

PROWSE, O, MP03-22

PSALLIDAS, E, MP01-06

PUGLISI, M, MP17-20

PULIATTI, S, MP04-13, MP16-11, MP26-02, MP27-17

PURI, A, V04-02, V17-07

PUROHIT, R, V11-07

PUSHKAREV, A, MP23-01, MP28-18

Q

QADRI, S, V10-01

QI, W, MP10-01, MP10-06

QIN, B, MP04-07

QING, W, BSP2-07

QU, X, MP06-05

QUADRINI, F, MP27-18

QUANLIANG, L., BSP1-15

QURAISHI, MM, MP02-09, MP05-15, MP06-19, MP14-02,

MP14-04, MP14-08, MP16-19, MP17-03, MP17-07,

MP18-12, MP20-10, MP22-05, MP26-08,

MP27-03, V11-12

QURESHI, H, MP30-18 
$\mathbf{R}$

R, V, V11-03

RADADIA, KD, MP19-21

RAFI, A, MP29-08

RAHEEM, A ABDEL, MP17-15

RAHIM, J, MP07-14, MP27-01, MP28-02, MP29-15

RAHMAN, M, MP07-21

RAI, A, MP20-09, MP21-14, MP24-19, MP28-16

RAI, B, MP02-04, MP02-21, MP20-19, MP24-10

RAI, S EL, MP04-02, MP14-18

RAISKY, J, MP20-07

RAISON, N, MP06-06, MP06-11, MP06-18, MP08-16, MP20-17

RAJAGOPALAN, A, MP16-19

RAJKUMAR, G, MP08-01, MP08-13

RAJU, S, MP27-10

RAJYAGURU, DA, MP05-12

RALPH, D, MP16-13

RAMACHANDRA, M, MP05-21, V01-02

RAMACHANDRAN, A., BSP1-20

RAMADAN, OA, MP05-15, MP16-19, MP26-08, MP27-03, MP30-01

RAMANATHAN, JM, V05-08

RAMASWAMI, K, MP03-16, MP11-12, V02-12, V07-06, V07-11, V07-12, V10-05

RAN, Q, MP06-04

RANDHAWA, K, MP13-16

RAO, P, MP24-11, V01-01, V10-11

RAPOPORT, L, MP01-04, MP04-14, MP10-17, MP30-05

RASHAD, H, MP16-09

RASHID, H, V03-11, V15-07

RASMUSSEN, R, MP14-11

RASTINEHAD, AR, MP02-01, MP14-16, MP14-22

RATANAPORNSOMPONG, W, MP12-21, V03-09

RATNANI, P, BSP1-17, MP02-01, MP19-01

RAVEENDRAN, R, MP03-16, MP11-12, V02-12, V07-06, V10-05

RAVEENDRAN, V, MP19-19

RAVINDRAANANDAN, M, MP03-05, MP21-02, MP22-10, MP29-05, V04-12, V07-01, V07-02

RAWANDALE-PATIL, A, MP06-01, MP06-12

RAWANDALE, PA, MP06-01, MP06-12

RAYNAL, G, MP27-05

RAZA, A, MP21-10, MP21-17

RAZVI, H, MP04-11, MP04-17, MP24-04

RAZZAK, O ABDEL, MP01-20

REASON, J, MP01-06

REDDY, N, MP26-20

REEVES, T, MP05-16, MP21-13, MP27-07, MP29-14

REGAN, D, MP10-20

REGIS, F, BSP1-16, MP01-02, MP16-20, MP19-20, V02-08

REIMERINK, JJ, MP04-03

REINHARD, R, MP04-03

REIS, R, MP16-08, V17-10

REIS, S, MP11-05, MP17-05

RENDON, H, MP28-04

RENSING, A, V06-01

RESAD, S, MP06-03

RESTREPO, J, MP05-01

REYNOLDS, LF, MP30-13

RHA, K, MP17-15, V09-05

RHAINDS, M, MP25-07

RIBEIRO, F, V16-04
RICE, P, MP30-10

RICHARDS, KA, MP06-02

RICHSTONE, L, MP15-23

RICO, L, MP14-05, MP26-06

RIDGWAY, AJ, MP02-18

RIGGS, D, BSP2-20

RIHAI, X, MP01-09, MP15-03, MP15-08, MP15-10, MP15-19, MP25-12, MP27-13, MP28-12

RIJO, E, MP03-18

RIMINGTON, PD, MP15-06, MP19-03

RING, J, MP22-20

RINGA, M, MP26-06

RIVAS, J GOMEZ, MP03-18, V07-03

RIVERA, ME, MP08-03, MP09-14, MP23-07, V18-04

RIVERO, A, MP24-06

RIZVI, A, MP30-18

RIZZO, G, MP16-05, V17-08

RIZZO, M, MP16-11

RIZZON, MV, V16-04

ROBERT, G, MP16-10, MP30-09

ROBERTS, J, V16-05

ROBERTS, WW, MP25-05, MP30-04, MP30-16

ROBLES, A, MP24-06

ROCCO, B, MP01-15, MP03-12, MP04-13, MP06-08, MP07-10, MP08-18, MP09-12, MP12-05, MP14-17,

MP16-11, MP21-03, MP25-13, MP26-02, MP27-17

ROCHE, J, MP03-12, MP14-19

ROCHESTER, M, MP03-09

RODRÍGUEZ, J. DÍEZ, BSP1-08

RODRIGUES, R, MP06-07, MP24-12, MP24-18, MP28-22

RODRIGUEZ, F MILLÁN, MP22-21, V04-04

RODRIGUEZ SOCARRAS, ME, MP02-13, MP03-18, MP26-21, V07-03, V18-10

ROEHRBORN, C, MP03-07, MP09-20

ROGERS, C, V02-07, V14-09

ROGERS, T, MP06-10, MP12-05, MP12-11, MP12-19, MP17-12, V03-02, V03-07

ROJAS, E, MP07-18

ROMERO, A, MP28-04

ROMSAITHONG, U, MP14-12

ROOF, S, MP12-11, MP12-19

ROSADO, J, MP27-02

ROSE, K.M., BSP1-09

ROSE, KM, MP18-09

ROSEN, D, MP18-18

ROSENBAUM, CM, MP29-03

ROSENBERG, L, MP20-13

ROSENBLUTH, E, MP23-14, MP23-15

ROTH, J, MP03-02

ROUPRËT, M, MP14-19

ROUPRET, M, MP15-23

ROY, A., BSP1-07

ROYO, F CASTROVIEJO, V01-12, V03-12, V12-05, V16-06

RUAN, Y, V07-10

RUBIO, DT, MP13-14, MP28-08

RUIQUAN, X, MP27-13

RUIZ, M CARLOS REINA, MP21-12

RUKIN, N, MP30-08

RUSSELL, D, MP06-09

RUSSO, N, MP11-17, V02-02

RUSSO, N., BSP1-18

RYOO, H, MP26-09 
S

SÁNCHEZ-MARTÍN, F, MP22-21, V04-04

SÁNCHEZ, H, MP04-19, MP07-18, MP22-22

SAAD, I, MP01-20, MP12-08

SAAD, TA Al, V06-05

SABIOTE, L, V04-04

SABNIS, R, BSP2-03, MP10-08, MP19-10, MP25-17, MP26-20, V15-05

SADHWANI, S, BSP2-19

SAEB-PARSY, K, MP21-01

SAEED, AA, MP29-18

SAFARALY, H, MP19-18

SAFIULLIN, R, BSP2-21, V08-07, V08-08

SAGEHASHI, R, MP18-20

SAHA, P, MP07-21

SAIFEE, Y, MP13-21

SAINI, J, MP17-21

SAITO, M, MP12-14, MP18-20, V14-11

SAITTA, G, MP02-13, MP03-18, MP23-12, MP26-21, V07-03, V18-10

SAJJAD, M., BSP1-19

SAKAI, H, MP12-15, V05-12

SAKAMOTO, N, MP12-17, MP13-13

SAKURAI, M, MP09-06

SALAM, B, MP28-01

SALEEMI, A, MP03-13

SALEM, S, V12-09

SALGIA, P, MP13-21

SALHI, Y. Al, BSP1-14

SALKINI, MW, BSP2-20, MP05-08, MP13-05, V15-08

SALONIA, A, MP05-06, MP29-19

SAMANIEGO, M. PASCUAL, BSP1-08

SAMSON, P, MP24-13

SAMSUDIN, A, MP05-03

SANDBERG, A, MP19-07

SANDERS, A, MP22-13

SANDKUYL, R, MP04-03

SANDRI, M, MP12-11

SANDS, K.G., BSP1-20

SANGARALINGAM, S, MP13-08, MP19-18

SANGUEDOLCE, F, MP01-02, MP16-20, V02-08

SANO, K, V17-02

SANO, M, MP09-06

SANTANA, TB, V11-03

SANTO, N, V17-02

SANTOS, HO, V11-03

SANTOS, O, V16-04

SARAN, R, MP27-19

SARAN, S, MP27-19

SARAOGI, M, MP05-21

SARCHI, L, MP16-11

SARHAN, O, BSP2-06, BSP2-13

SARI, I, MP24-21

SARICA, K, MP06-06, MP10-03

SARKAR, S, MP08-01, MP08-13

SARKIS, Á, MP15-20

SARMA, D, V16-12

SASAKI, J, MP29-01

SASSA, N, MP01-05, MP01-11

SATO, H, MP12-14

SATO, M, MP12-10

SATOH, S, MP18-20, V14-11

SAULAT, S, V10-01

SAUNDERS, L, MP22-18
SAURABH, C, MP28-13, MP30-02

SAURAV, K, V05-04, V12-02, V16-03

SAWADA, N, MP13-17

SAWANGCHAREON, A, MP16-07

SAWCZYN, GV, MP15-13, V09-08, V09-10, V13-07, V13-12

SAYEDAHMED, K, MP21-11

SAYEGH, C, MP02-03, MP24-15

SAZAWA, A, V10-03

SCARFO', F, MP26-21

SCARPA, R, V16-07

SCHAKAKI, S, V15-02

SCHIPS, L, MP02-13

SCHOLZ, D, MP11-15

SCHOOFS, F, MP10-02, MP16-01, V02-05

SCHULSINGER, D, MP24-05

SCHULSTER, ML, V11-06

SCHWAAB, T, MP01-01

SCHWALB, J FERNÁNDEZ-CONCHA, MP21-12

SCHWARTZ, BF, V18-01, V18-05

SCHWARTZ, JA, MP14-16

SCHWARTZ, M, MP20-03

SCOFFONE, C, MP07-04

SCOTLAND, K, MP04-20, MP10-07, MP23-07

SCOTT, C, MP06-17

SEBAALY, R, MP13-18

SEBESTA, E, MP24-15

SEE, KJ, MP01-10, MP17-16

SEIFERT, H, MP03-02, V11-11

SEKIHARA, T, MP26-10

SEKINE, Y, MP03-17

SELCUK, B, V08-05, V08-06

SELTZER, R, MP27-12, MP27-16

SEMINS, M, MP07-19

SEN, U, MP17-09

SENER, T, MP07-04, MP24-21, MP28-11, MP30-08

SEO, H, MP19-11

SEO, I, MP29-11

SEOANE, L MOSQUERA, V02-08

SEOW, C, MP04-20

SEPAHA, A, MP13-21

SERNI, S, MP18-08

SESMERO, J H. AMÓN, MP21-12

SETIA, S, MP05-05, V04-05

SETO, K, V02-03, V04-01

SEVERGINA, L, MP01-04

SFAKIANOS, J, MP02-19, MP14-22, MP18-05, MP18-18, V17-05

SHADPOUR, P, MP24-08

SHAFIEI, S BESHARAT, MP06-15

SHAH, A, MP27-09

SHAH, M, MP04-08, MP21-05, MP21-22, MP24-07, MP30-11

SHAH, O, MP02-03, MP22-14, MP24-15

SHAH, S, MP21-01

SHAHAIT, M, MP01-13, MP09-17, MP12-04,

MP19-07, MP20-20, V11-01

SHAHROUR, W, MP03-22, V07-04

SHAHZAD, A, MP18-10

SHARIFIAN-AMIRI, R, MP13-12

SHARMA, A, MP05-05

SHAUN, W, MP02-15

SHAW, EJ, MP17-18

SHAYEGAN, B, MP06-09, MP17-21, MP22-06, MP22-17

SHAZLY, M El, MP21-08, V07-08 
SHEIKH, M, MP02-09

SHERGILL, I, BSP2-02

SHERGILL, IS, BSP1-05, MP03-13

SHERIF, E El, MP03-01, MP21-08

SHEYNKIN, Y, MP24-05

SHI, B, MP24-16

SHIBATA, Y, MP03-17

SHIDA, Y, MP12-15, V05-12

SHIGA, K, MP12-16

SHIGEMURA, K, MP19-22

SHIM, M, MP02-11

SHIMBO, M, MP09-06, MP12-02

SHIMIZU, H, MP03-11

SHIMIZU, R, MP13-13

SHIMURA, S, MP16-02

SHIN, S, MP09-08

SHIN, T, MP18-06

SHIN, Y, MP27-11

SHINOHARA, N, V10-03

SHIRAISHI, Y, MP12-06

SHIROKI, R, MP15-16

SHIROKI, R., BSP1-03

SHIROTAKE, S, MP15-02, V16-02, V17-02

SHMANEV, A, V16-08

SHOJO, K, V14-04

SHROTRI, N, MP05-15, MP16-19, MP20-10, MP20-15, MP26-08, MP27-03, MP30-01

SHUKLA, S, MP23-22

SHUMATE, A, MP17-06

SHUT, E., BSP1-21

SIAM, A, MP15-09

SIERRA, A, MP14-10, V10-06, V18-02, V18-11

SIGHINOLFI, M, MP01-15, MP03-12, MP04-13, MP06-08, MP07-10, MP08-18, MP09-12, MP14-17, MP16-11, MP21-03, MP25-13, MP26-02, MP27-17

SILVA-RAMOS, M, MP18-04

SILVA, CM, MP17-04, MP17-05

SILVA, MV, MP28-05

SIM, A, MP03-20, MP06-14, V02-01

SIMEONE, C, MP13-01, MP13-09

SIMOES, A, MP05-15, MP16-19, MP20-10, MP20-15, MP27-03

SIMONE, G, MP09-05, MP11-20, MP13-01, MP13-09, MP14-19, MP15-15, MP15-18, MP17-20,

MP18-19, MP18-21, V08-11, V14-06

SIMONYAN, D, MP25-14

SIMSEKOGLU, M, V14-03

SINGER, EA, MP19-21

SINGH, A, BSP2-03, MP10-08, MP11-22, MP19-10, MP25-17, MP26-20, V15-05

SINGH, P, BSP1-07, MP02-12

SINGH, V, MP08-17, MP15-04

SINHA, L, MP24-11

SINHA, RJ, MP08-17, MP15-04

SIVALINGAM, S, MP05-14

SIVARAMAN, A, BSP1-20, MP06-22, MP11-11, MP19-13

SIVKOV, A., BSP1-21

SKOLARIKOS, A, MP05-16, MP10-03, MP30-08

SLABA, S, MP23-19

SLAWIN, JR, V13-03

SMAYRA, T, MP23-19

SMITH, AD, MP20-09, MP21-14, MP24-14, MP24-19, MP28-16, V04-08, V18-03

SMITH, B, MP16-14
SMITH, J, MP04-08, MP09-10, MP14-06, MP21-22

SMITH, JC, MP09-03

SMITH, M, MP29-10

SMITH, R, MP05-09

SMYTH, N, MP07-08

SNELL, J, MP14-20

SNETSELAAR, L, MP22-19

SNIADECKI, K, MP20-08

SOBEL, DW, MP20-18, MP24-17, MP26-01

SOBOTKA, S, MP19-01

SOFER, M, MP07-04

SOLANO, C, MP05-01

SOMANI, BK, MP05-16, MP05-21, MP06-13, MP06-17, MP10-03, MP11-16, MP14-07, MP14-21, MP16-06, MP21-13, MP24-10, MP27-07, MP27-15, MP29-14,

MP29-21, MP30-08, MP30-10, MP30-17, V01-02, V04-10 SONG, M, MP19-11

SONG, P, MP26-12

SONI, R, V18-09

SOOMRO, A, MP23-11, V10-01

SORENSEN, M, MP06-16

SORIANO-MORILLO, D, V10-09

SOROKIN, I, MP29-16

SOROKIN, N, MP01-04

SOTO RODRÍGUEZ, J, BSP1-08, MP16-22, V17-03

SOUSA, AL, MP17-04, MP17-05

SRINUALNAD, S, MP02-22, MP17-08

SRIPRASAD, S, MP29-10, V05-07, V18-08

SROUGI, M, MP11-05, MP13-22, MP17-04, V12-08

SROUGI, V, MP13-22, V12-08

STAMBAKIO, H, MP30-14

STAN, M, MP25-21

STANOWSK, M, MP17-07

STANOWSKI, M, MP14-04, MP14-08, MP14-15, MP17-03, V11-12

STANTON, ML, MP09-04

STARMER, B, MP05-03

STEINBERG, RL, MP06-05, MP09-20, MP13-10, MP14-11, MP22-09, MP22-11, MP22-16, MP29-01, MP29-16, V09-07, V13-01

STEPHENS, C, V01-03

STERLING, J, MP19-21

STERN, K, MP07-04

STERN, M, V03-03, V08-10, V13-09

STERN, N, MP04-11

STERNBERG, K, MP24-17

STIFELMAN, MD, BSP2-15, MP10-21, MP11-01, MP14-14, MP18-02, MP18-13, MP18-17, MP18-18, V13-10, V15-04

STOECKLE, M, MP15-23

STOIANOVICI, D, MP10-11

STOKES, P, MP04-08, MP21-05, MP21-22, MP24-07, MP30-11

STOLLER, M, MP20-05, MP23-18, V05-05

STONE, Y, V02-10

STOUGHTON, C, MP10-04

STRAUSS, D, MP09-19, MP17-10

STREEPER, NM, MP05-14, MP22-13

STREETER, EH, MP06-19, MP14-02, MP14-04, MP14-08, MP14-15, MP15-06, MP16-19, MP17-03, MP19-03, MP20-10, MP20-15

STRUCK, JP, MP01-18, MP29-06

STUBINSKI, R, BSP2-16, MP03-04

STUTZ, J, MP09-03, MP09-10 
SU, B, MP23-18

SU, H, MP17-16

SUBIELA, J, MP19-20

SUBRAMANIAM, S, MP27-03

SUCUPIRA, DG, V12-08

SUGI, M, MP21-18

SUGINO, Y, MP12-01, MP13-20, MP16-04

SUJENTHIRAN, A, MP03-08

SULAIMAN, S, MP16-06

SULLIVAN, B, MP01-01

SULTAN, G, MP30-18

SULTAN, M, MP03-01

SUN, J, MP27-04

SUN, Q, MP04-21

SUNDARAM, C, MP13-09

SUNG, H, MP21-15

SUNG, J, MP09-03, MP09-10, MP11-08, MP14-01, MP16-03, MP21-07, V02-11 SUNGKUM, P, MP12-21, V03-09

SUR, RL, MP05-14, MP10-04, MP10-08, MP26-11 SUYAMA, T, V03-04, V05-02

SUZUKI, I, MP12-01, MP13-20, MP16-04, MP17-17

SUZUKI, K, MP03-17, MP26-10

SUZUKI, R, MP17-17

SVINDLAND, A, MP12-22

SWAMY, G KANDA, MP02-04, MP02-21, MP20-19

SWINN, M, MP15-06

SYAL, A, MP18-09

SYMES, A, MP24-22

SYUTO, T, MP03-17

\section{T}

TABACZYNSKI, W.A., BSP1-19

TABAKIN, A, MP19-21

TABATA, K, MP12-09, MP16-02

TABCHE, A, MP23-19

TABIB, C, MP02-14, MP20-03, MP20-09, MP24-13, MP24-14, V04-08, V18-03

TABREZ, S, V11-09

TACCOEN, X, V03-01, V16-11

TAGUCHI, M, MP21-18

TAHRA, A, MP17-09

TAI, M, MP13-04

TAILLY, T, MP05-16, MP30-08

TAK, GR, MP19-10

TAKAHARA, K., BSP1-03

TAKAHASHI, D, V02-03, V04-01

TAKAHASHI, K, MP03-11

TAKAHASHI, N, MP13-17

TAKAHASHI, T, V17-02

TAKAZAWA, R, MP25-01

TAKEDA, H, MP03-11, MP05-19

TAKEDA, M, MP13-17

TAKENAKA, A, MP13-13, MP15-16

TAKENAKA, M., BSP1-03

TALAMINI, SM, MP17-19

TALANKI, V, MP24-05

TALCOTTT, M, MP11-11

TALSO, M, MP30-08

TAM, C, MP20-07

TAMIM, H, MP08-14, MP12-03, MP13-18

TAMISETTI, V, MP14-16

TAN, D, V10-04
TANAKA, M, MP11-13

TANG, J, MP22-19

TANG, W, MP29-20

TANG, Y, MP07-16, MP20-05, MP22-03

TANGUAY, S, BSP2-05

TANIDIR, Y, MP07-04, MP24-21, MP25-06, MP28-11

TANURE, L, MP15-20

TANWAR, H, MP07-06

TAOKA, Y, MP16-02

TAOULI, BA, MP14-16, MP14-22

TAPIERO, S, MP05-14, MP10-20, MP11-07, MP11-08, MP11-14, MP14-01, MP16-03, MP20-11, MP21-07

TARAZONA, N, MP05-01

TARICIOTTI, P, MP16-05, V17-08

TASIAN, GE, MP30-14

TATARANO, S, MP15-22

TAUBER, S, MP20-08

TAVARES, C, MP18-04

TAWEEMONKONGSAP, T, MP02-22, MP14-12, MP17-08

TAWFIEK, ER, MP10-07

TAYEB, M El, MP09-03, MP09-10

TEFIK, T, MP24-09

TEICHMAN, J, MP30-04

TEMA, G, V17-08

TERAOKA, S, MP13-13

TERRITO, A, BSP1-16, MP01-02, MP16-20, MP18-08, MP19-20, V02-08

TERRY, RS, MP04-22, MP21-20

TEWARI, A, BSP1-02, BSP1-17, MP02-01, MP19-01, V17-05

THAI, J, MP05-10

THAI, KH, MP09-03

THAKARE, N, MP22-18

THEIL, G, MP19-20

THIEL, D, MP06-10, MP19-08

THIRUCHELVAM, N, MP03-09

THOMAS, JA, MP14-20

THOMAS, K, MP20-19

THOMAS, M, MP06-19, MP16-19, MP18-12, MP20-10, MP20-15

THOMAS, R, MP17-18

THOMAZ, E, MP22-13

THOMPSON, J, MP08-12

THURAIRAJA, R, MP15-06

THURMON, KL, MP03-19, MP08-12, MP08-15, V07-05

THWAINI, A, MP13-16

TIAN, Y, MP04-01

TIANPENG, X, BSP1-15, MP15-03

TIMUROGLU, B, MP24-21

TIONG, HY, MP03-20

TOCHIGI, K, MP01-05, MP01-11

TOENNIESSEN, AM, MP20-08

TOFFEQ, HM, MP07-02, MP29-18

TOHI, Y, MP12-01, MP13-20, MP16-04, MP17-17

TOLOFARI, S, MP05-03

TOMITA, K, V05-10

TORRECILLA, C, MP23-16

TORRECILLA, J, V01-12, V03-12, V12-05, V16-06

TORRES, J, MP01-19, MP06-07, MP24-12, MP24-18, MP28-22

TORRES, JE, MP04-19, MP07-18, MP22-22

TOSCO, L, MP08-09

TOWE, M, MP01-12, MP17-16

TOYAMA, Y, MP03-11

TRACEY, A, V09-02, V15-11 
TRACY, C, MP22-09, MP22-11, MP22-16

TRAN, H, MP05-04

TRAN, J, MP01-10, MP17-16

TRAXER, O, MP04-06, MP04-09, MP05-22, MP10-14, MP16-10, MP20-06, MP21-21, MP25-09, MP27-05, MP29-12, MP29-19

TREACY, P, BSP1-02, BSP1-17, MP02-01, MP14-22, MP28-14, V17-05

TRIVEDI, H, MP09-20

TROTTER, M, MP02-20

TRUONG, H, MP27-02

TSAI, J, MP26-15

TSAI, L, V09-01, V16-09

TSAO, Y, MP23-06

TSARICHENKO, D, MP04-14, MP10-17, MP30-05

TSE, J, MP29-01

TSENG, J, V07-09

TSOUNAPI, P, MP13-13

TSUI, JF, MP10-21, MP11-01, V03-05, V13-08, V14-08

TSUJII, T, MP25-01

TSUJIOKA, T, V02-03, V04-01

TSUKUDA, F, MP12-17

TSUMURA, H, MP12-09

TSUTSUMI, N, MP17-17

TSUZUKI, T, MP12-18

T.P., R, V16-12

TUBARO, A., BSP1-14

TUCCI, C, MP20-18, MP26-01

TUDERTI, G, MP09-05, MP11-20, MP14-19, MP15-15,

MP15-18, MP17-20, MP18-19, MP18-21, V08-11, V14-06

TUFEK, İ, MP16-17, MP18-15

TUGCU, V, MP18-08

TUNA, MB, MP16-17, MP18-15

TUNC, L, MP02-06, MP08-20, V01-07

TUNCEL, A, MP08-20, MP15-07

TURAN, S, MP24-09

TURKMEN, N, BSP2-10, BSP2-17, MP03-03

TUZZOLO, P, V16-07

TYAGI, V, V05-04, V12-02, V16-03

TZAVARAS, N, BSP1-02, V17-05

$\mathbf{U}$

UEDA, T, MP05-19

ULKER, V, MP25-20

UMER, D, MP30-20

UMEZ-ERONINI, NO, MP11-18, MP14-07

UMEZAWA, Y, V17-02

URMANTSEV, M, V08-07, V08-08

UTSUNOMIYA, T, MP16-02

UY, M, MP22-06, MP22-17

UZZO, R, MP13-09

$\mathrm{V}$

VADDI, C, V10-07, V12-04

VAGIONIS, A, MP23-13, MP26-03, MP30-03

VALADON, C, MP05-13, MP28-07, MP30-15

VALERO, RJ, V09-10

VAN BASTEN, J, MP19-09

VAN CLEYNENBREUGEL, B, MP06-13, MP06-17, MP11-16

VAN DER BERG, E, BSP2-19

VAN DER POEL, H, MP19-09

VAN DER VIJGH, RK, MP04-03
VAN HAARST, EP, MP04-03

VAN MERRIËNBOER, J, MP19-09

VANACORE, D, BSP1-16, MP01-02, MP16-20, MP18-08, MP19-20, V02-08

VARGA, B, MP16-19

VARMA, R., BSP1-12

VASDEV, N, MP15-06

VASQUEZ, A, MP05-01

VASUDEVAN, V, MP02-14, MP21-14, MP24-14, V04-08

VAVASSORI, I, MP08-21, V01-10

VECCIA, A, MP13-01, MP13-09, MP18-21, V09-02,

V11-05, V15-11

VEER, S, MP24-11, V01-01, V10-11

VELIZ-CABRERA, G, MP07-20

VENENZIANO, D, MP06-13, MP06-17, MP11-16

VENKATESH, R, BSP1-20, MP06-22, MP11-11, MP16-12, MP18-16, MP19-13, MP22-12, V14-01

VENTIMIGLIA, E, MP05-22, MP10-14, MP20-06, MP27-05, MP29-19

VERMIGLIO, M, MP16-05

VERSOS, R, MP18-04

VERZOTTI, G, MP25-09, MP30-09

VETTER, J, MP06-22, MP16-12, MP18-16, MP19-13, MP22-12

VICENTINI, FC, MP04-19, MP07-04, MP07-18

VIG, A, MP24-02

VIGUES, F, MP23-16

VILLA, L, MP05-06, MP20-06, MP29-19

VILLALBA, A ALONSO, BSP1-08, V01-12, V03-12, V12-05, V16-06

VILLASANTE, N, MP08-19

VILLEGAS, P, MP05-01

VIPRAKASIT, DP, MP05-14

VISSAMSETTI, B, MP02-16

VITAGLIANO, G, MP14-05

VON ROHDEN, E, MP11-15

VRANIS, NM, V11-06

W

WACTAWSKI-WENDE, J, MP22-19

WADA, K, MP21-09

WAGENIUS, M, MP07-15

WAGH, Y, MP13-08, MP19-05

WAGNER, A, MP15-23

WAGNER, C, MP19-09

WAJSWOL, E, BSP1-02, MP02-01, MP14-14, MP14-16, MP14-22, V17-05

WALL, F, MP14-21

WALTER, PJ, MP02-20

WALTERS, U, MP09-01

WANG, D, MP02-15, MP03-21, MP06-03, MP21-04

WANG, G, MP12-12, MP13-03, V03-08, V15-09

WANG, K, BSP2-04, BSP2-18, MP01-21, MP04-21, MP09-22, MP19-14

WANG, L, MP02-15, MP04-20

WANG, Q, MP04-07, MP10-10, MP10-18, MP23-04, MP28-15

WANG, S, BSP2-07, MP04-07, MP07-16, MP10-10, MP10-18, MP22-03, MP23-04, MP28-15

WANG, W, MP04-01, MP23-08

WANG, Y, MP06-09

WARLOW, A, MP14-20

WASEDA, Y, MP25-01 
WASON, S, MP03-21, MP06-03, MP20-13, MP21-04, V01-09

WATANABE, K, V17-02

WATANABE, S, V14-04

WATSON, M, MP11-22

WATSON, R., BSP1-19

WEI, H, V12-03

WEI, Q, MP01-07

WEI, T, MP13-04

WEI, Y, BSP2-09, BSP2-14, V12-01

WEIL, R, MP02-01, MP19-01

WEIMERSHEIMER, $\mathrm{P}, \mathrm{MP} 24-17$

WEISS, RE, MP19-21

WELTER, L, V11-03

WEN, X, MP22-19

WENZENG, Y, MP10-15, MP25-16

WESSELLS, H, MP06-16

WETTERAUER, C, MP03-02, V11-11

WHELAN, P, MP04-22, MP21-20

WHILES, BB, MP03-19, MP08-12, MP08-15, V07-05

WHITE, MD, MP24-02

WHITEHURST, L, MP21-13

WHITING, D, MP01-17, MP29-10, V05-07, V18-08

WIDMER, A, MP03-02

WIEBMEYER, JR, MP22-20

WIENER, S, MP23-18

WIJBURG, C, MP15-23

WIKLUND, P, BSP1-02, MP02-01, MP15-23, MP19-01, V17-05

WILLIAMS, DH, MP06-02

WILLIAMS, J, MP04-12, MP09-18

WILLINK, M Tjeenk, MP04-03

WILSON, I, MP14-21

WINOKER, JS, MP04-15, MP14-16, MP14-22

WINSHIP, B, MP04-22, MP21-20

WISEMAN, O, MP21-01, MP21-21

WISSING, M, BSP2-05

WITHINGTON, J, MP23-16

WITTHAUS, M, V03-11

WOLLIN, D, MP04-22

WONG, NC, MP06-09

WOO, J, MP09-16

WOOD, H, V13-04

WOOD, K, MP04-18, MP22-04, MP22-07

WOODHAMS, S, MP15-06

WOODWARD, A, MP22-06

WORANISARAKUL, V, MP02-22, MP14-12, MP17-08

WRIGHT, M, MP19-03

WU, G, V03-11, V06-03, V15-01, V15-07

WU, H, V09-01

WU, J, BSP2-09, BSP2-14, MP13-15, V09-06, V12-01, V16-01

WU, W, BSP2-09, MP23-03, MP24-13, MP25-22

WU, Y, MP02-08, MP04-07, MP10-10, MP10-18, MP23-04, MP28-15

WÜLFING, C, MP13-02, V05-03, V10-02

$\mathbf{X}$

XAVIER, G, MP11-05

XAVIER, MM, MP11-05, MP17-04, MP17-05

XI, Y, MP14-11

XIA, S, V07-10

XIAO, B, MP07-16, MP22-03, MP23-18

XIAO, K, BSP2-04, BSP2-18
XIAOFENG, Z, BSP1-15, MP01-09, MP15-03, MP15-08, MP15-10, MP15-19, MP25-12, MP27-13, MP28-12

XIAONING, W, MP15-08, MP15-10, MP15-19, MP25-12, MP28-12

XIAOQIANG, S, MP10-15

XIN, Z, MP01-09

XIONG, L, MP07-11, MP07-12, MP11-10

XIONG, W, MP06-04, V14-12

$\mathrm{XU}, \mathrm{B}, \mathrm{V} 16-01$

XU, K, MP16-18

XU, Q, BSP1-22, MP04-16, MP05-18, MP07-11, MP07-12, MP28-03

$\mathrm{XU}, \mathrm{R}, \mathrm{MP} 11-22$

XU, Y, V02-13, V04-11

XUN, Y, MP23-04, MP28-15

\section{Y}

YAACOUB, R YOUSSEF, MP20-11, MP21-07

YABUSAKI, R, MP12-06

YADAV, S, V05-04, V12-02, V16-03

YAFI, F, MP01-12, MP17-16

YAGHOUBIAN, A, MP23-12

YALCIN, S, MP02-06, V01-07

YAMAGUCHI, A, MP21-09

YAMAGUCHI, R, MP17-17

YAMAMOTO, A, MP13-13

YAMAMOTO, H, MP12-20, MP17-13

YAMAMOTO, S, MP21-09

YAMAMOTO, T, MP01-05, MP01-11

YAMANAKA, T, V17-02

YAMANAMA, M, V02-03, V04-01

YAMAUCHI, Y, MP02-07

YAMEOGO, C, BSP2-16, MP03-04

YAN, R, MP23-07

YANAGI, M, MP03-11

YANG, C, V09-01

YANG, J, MP06-04, MP17-01

YANG, L., BSP1-20

YANG, T, MP26-19

YANG, X, MP01-07

YANG, Z, MP13-15

YANQIAO, Z, MP10-15, MP25-16

YAO, H, MP05-04

YAO, M, BSP2-11

YAO, R., BSP1-19

YASIN, J, MP04-10

YASUDA, M, V17-02

YAU, R, V03-03

YAU, RJ, MP15-13, V09-08, V09-10, V13-07, V13-12

YAVUZSAN, A, BSP2-10, BSP2-17, MP03-03, MP23-02

YAZICI, C, MP25-06

YE, D, MP09-22, MP19-14

YE, H, MP07-12

YE, L, MP13-15, V12-01

YESILDAL, C, BSP2-10, BSP2-17, MP03-03, MP23-02

YILMAZ, S, MP02-06, V01-07

YODKHUNNATHAM, N, MP17-08

YOGESWARAN, C, V16-05

YOKOO, T, MP14-11

YOKOMIZO, A, MP12-16

YONEMORI, M, MP15-22

YONEYAMA, T, MP12-20, MP15-14, MP17-13

YONG, PC, MP02-05, MP19-04 
YOO, J, MP23-05

YOO, T, MP09-08

YOON, R, MP16-03

YOON, Y, V09-05

YÖRUBULUT, M, MP02-06

YOSHIDA, K, MP21-18

YOSHIDA, S, MP25-01

YOSHIDA, T, MP15-17, V05-10

YOSHIMURA, K, MP12-06

YOSHINO, H, MP15-22

YOU, D, MP18-01, V06-04

YOU, H, MP14-06

YOU, J, MP27-11

YOUNES, E, V15-12

YOUSSEF, PM, BSP2-15, MP18-02, MP18-13, MP18-17, V13-10, V15-04

YU, C, MP02-17, MP18-14, MP26-15

YU, J, MP02-10

YUANHU, Y, MP15-03, MP15-08, MP15-10, MP15-19, MP25-12, MP27-13, MP28-12

YUH, B, MP15-23

$\mathbf{Z}$

ZAHID, R, MP13-06

ZAHRAN, A, MP16-09, MP29-22

ZAKARIA, A, BSP2-05

ZAM, N MOHD, V04-07

ZAMPINI, AM, MP04-04, MP05-07, MP05-10, MP22-01, MP23-09, MP23-14, MP23-15, MP30-12, MP30-22

ZANATY, F, MP21-08
ZANETTINI, AC, V16-04

ZANETTINI, LA, V16-04

ZANG, C, MP13-01, MP13-09

ZARGAR, H, MP13-22

ZARKA, J, MP12-03

ZHAN, X, V02-13, V04-11

ZHANG, C, MP12-12, V03-08

ZHANG, F, BSP1-22, MP07-11

ZHANG, H, V02-13, V04-11

ZHANG, J, MP10-18

ZHANG, L, V09-06, V16-01

ZHANG, M, V03-03, V09-12

ZHANG, R, BSP2-09

ZHANG, X, MP07-16, MP22-03

ZHANG, Y, MP22-03

ZHANG, Z, MP04-16

ZHAO, F, V07-10

ZHAO, LC, V11-06, V13-03, V13-05

ZHIHUA, H., BSP1-15

ZHONG, L, MP27-04

ZHONG, P, MP04-22

ZHOU, L, MP01-21, MP04-21

ZHOU, X, MP12-12, MP13-03, V03-08, V15-09

ZHU, X, MP07-03

ZHU, Y, MP01-21, V07-10

ZHU, Z., BSP1-22

ZIEMBA, JB, MP30-14

ZIPUNNIKOV, V, V06-08, V16-08

ZLATOPOLSKY, A, MP24-05

ZORN, K, MP03-10

ZU, Q, MP13-15 